\title{
Wireless Instrumented RB Experiment Preliminary Design and Analysis
}

Approved for public release. Distribution is unlimited.
Padhraic L. Mulligan Kurt Smith

N. Dianne B. Ezell Daniel C. Sweeney Kara Godsey Adam James Annabelle Le Coq Joel McDuffee Shawn Stafford Jeffrey Arndt Jorge Carvajal Christian M. Petrie

December 2020 


\section{DOCUMENT AVAILABILITY}

Reports produced after January 1, 1996, are generally available free via US Department of Energy (DOE) SciTech Connect.

Website www.osti.gov

Reports produced before January 1, 1996, may be purchased by members of the public from the following source:

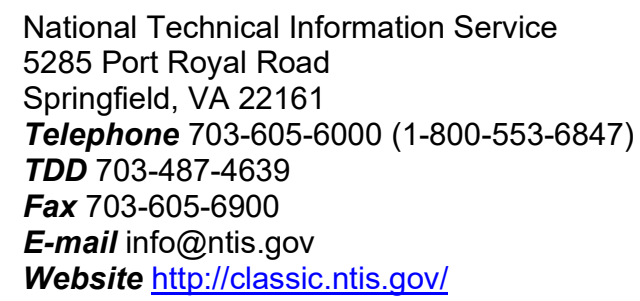

Reports are available to DOE employees, DOE contractors, Energy Technology Data Exchange representatives, and International Nuclear Information System representatives from the following source:

Office of Scientific and Technical Information

PO Box 62

Oak Ridge, TN 37831

Telephone 865-576-8401

Fax 865-576-5728

E-mail reports@osti.gov

Website http://www.osti.gov/contact.html

This report was prepared as an account of work sponsored by an agency of the United States Government. Neither the United States Government nor any agency thereof, nor any of their employees, makes any warranty, express or implied, or assumes any legal liability or responsibility for the accuracy, completeness, or usefulness of any information, apparatus, product, or process disclosed, or represents that its use would not infringe privately owned rights. Reference herein to any specific commercial product, process, or service by trade name, trademark, manufacturer, or otherwise, does not necessarily constitute or imply its endorsement, recommendation, or favoring by the United States Government or any agency thereof. The views and opinions of authors expressed herein do not necessarily state or reflect those of the United States Government or any agency thereof. 
Nuclear Energy and Fuel Cycle Division

\title{
WIRELESS INSTRUMENTED RB EXPERIMENT PRELIMINARY DESIGN AND ANALYSIS
}

\author{
Padhraic L. Mulligan \\ Kurt Smith \\ N. Dianne B. Ezell \\ Daniel C. Sweeney \\ Kara Godsey \\ Adam James \\ Annabelle Le Coq \\ Joel McDuffee \\ Shawn Stafford \\ Jeffrey Arndt \\ Jorge Carvajal \\ Christian M. Petrie
}

Date Published: December 2020

Milestone \#: M3UF-21OR0212142

Prepared by

OAK RIDGE NATIONAL LABORATORY

Oak Ridge, TN 37831-6283

managed by

UT-BATTELLE, LLC

for the

US DEPARTMENT OF ENERGY

under contract DE-AC05-00OR22725 



\section{CONTENTS}

CONTENTS

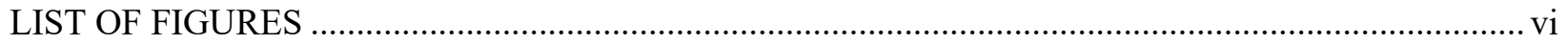

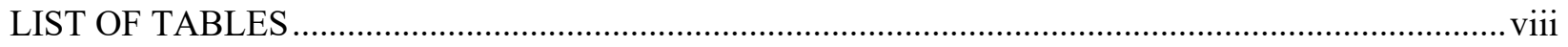

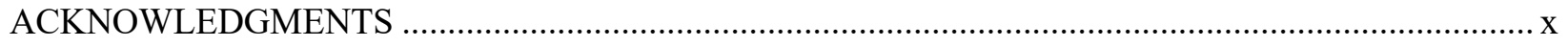

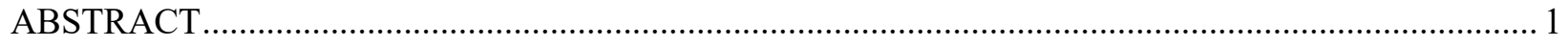

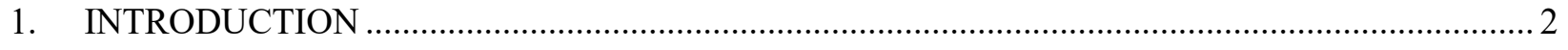

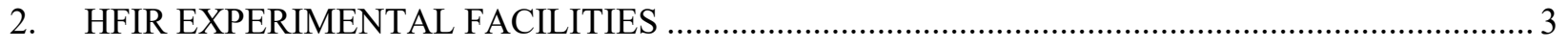

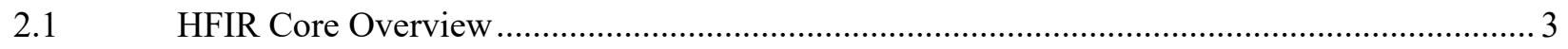

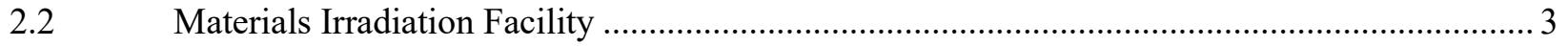

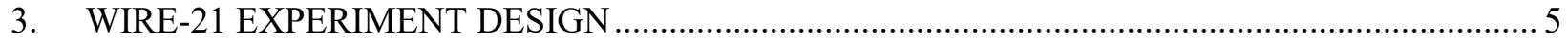

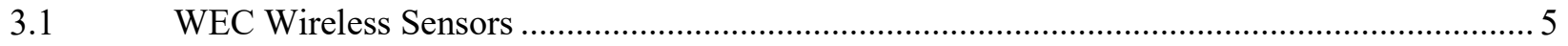

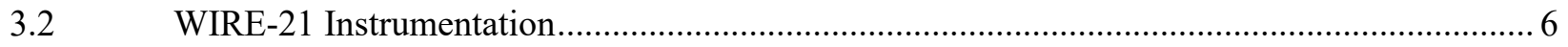

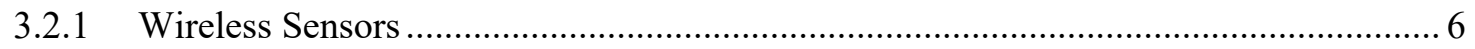

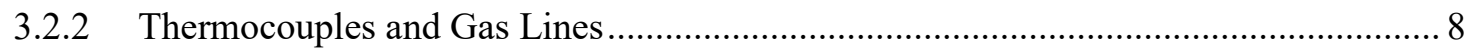

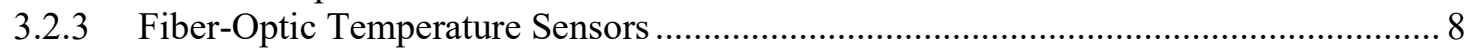

3.2.4 Self-Powered Neutron Detectors .................................................................. 8

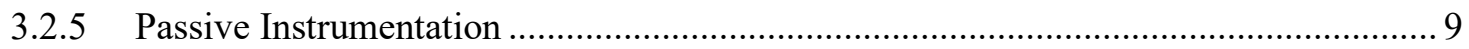

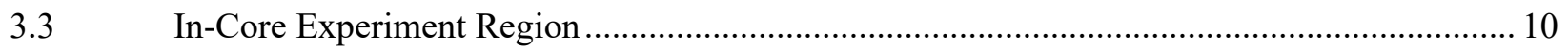

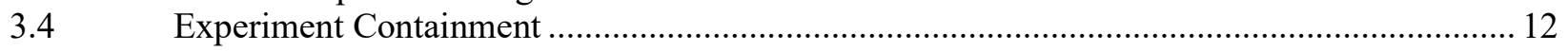

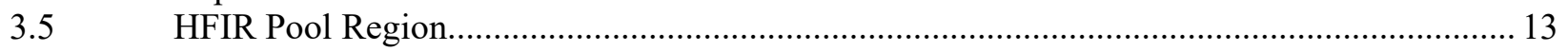

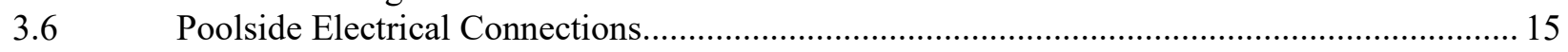

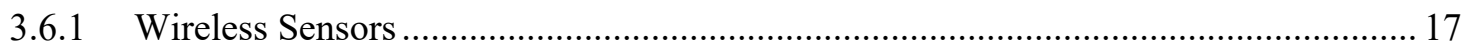

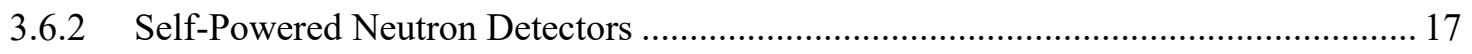

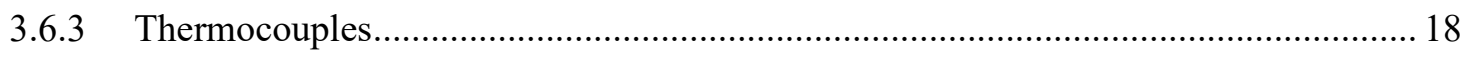

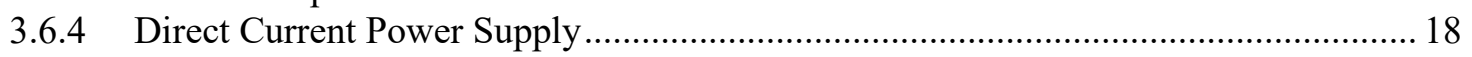

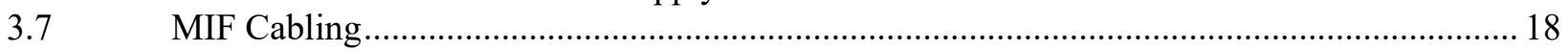

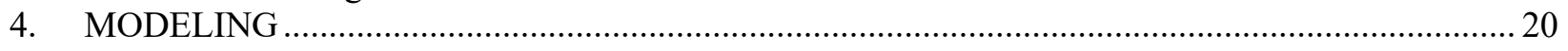

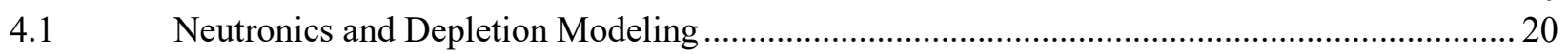

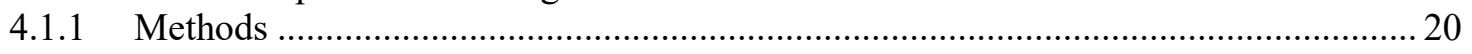

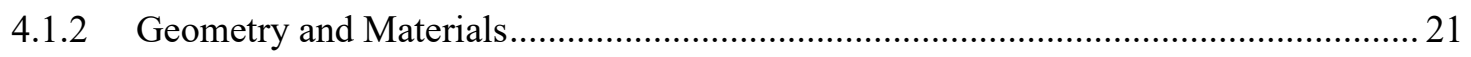

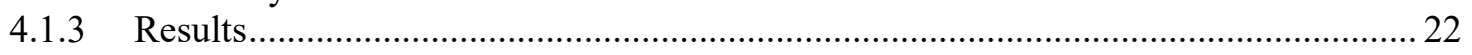

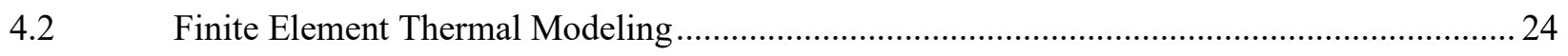

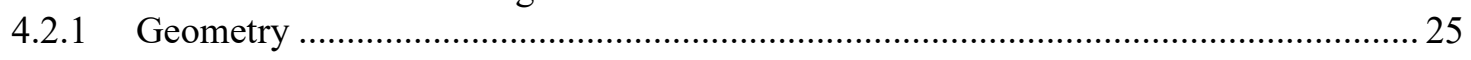

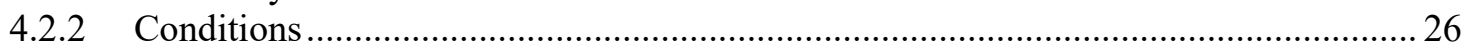

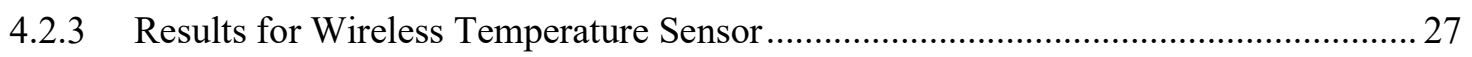

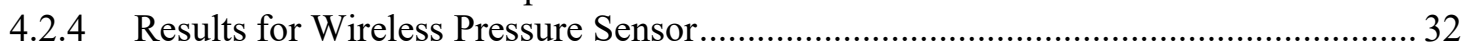

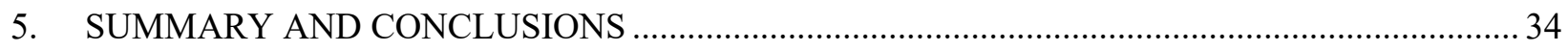

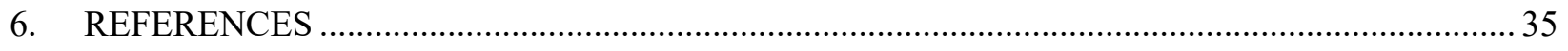




\section{LIST OF FIGURES}

Figure 1. Schematic cross-section of HFIR showing experimental positions.......................................... 3

Figure 2. Section views showing WEC's wireless temperature and pressure sensors............................... 5

Figure 3. In-core active instrumentation layout for the WIRE-21 experiment (not to scale)..................... 7

Figure 4. Electrical schematic for the SPNDs that will be used for real-time neutron flux monitoring

Figure 5. In-core region of the WIRE-21 experiment, with detail views showing the temperature and pressure sensor holders.

Figure 6. Instrumentation and gas line layout for three primary experiment locations.

Figure 7. WIRE-21 experiment containment showing the entire length and section views of specific regions.

Figure 8. Cross-sectional view of sensor leads and gas lines passing through the spiral channels in the shield plug.

Figure 9. Y-adaptor for splitting the experiment gas lines from the rest of the sensor leads.

Figure 10. Schematic showing routing of sensor leads within the HFIR pool from the top of the experiment's containment to the electrical junction box.

Figure 11: Cabling schematic for the experiment junction box.

Figure 12: WEC junction box with and without its lid. Internal components are not shown................... 17

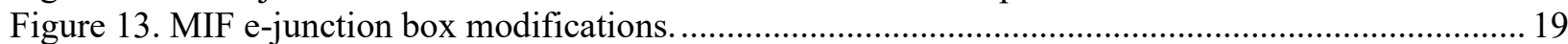

Figure 14. Cable routing between the HFIR pool-top and MIF lab space........................................... 19

Figure 15. Geometry used for radiation transport calculations.............................................................. 22

Figure 16. Heat generation rates in stainless steel vs. axial location for various days during a HFIR cycle. Dashed lines represent position of peak heat generation rate for day of cycle.

Figure 17. Geometry used for the finite element thermal modeling of WEC's wireless temperature and pressure sensors inside an experiment holder and housing, with numbering conventions for each inductor.

Figure 18. Cases assuming 98\% helium for a range of FS gas gaps and $\mathrm{min} / \mathrm{max}$ core peaking factors.

Figure 19. Cases run with $75 \%$ and $50 \%$ helium for a $0.24 \mathrm{~mm}$ FS gas gap and $\mathrm{min} / \mathrm{max}$ core peaking factors.

Figure 20. Inductor temperatures for several sensor positions above and below the midplane, with the position of the top of the sensor, relative to the midplane, indicated in the figure legend.

Figure 21. Inductor temperatures for several holder-to-housing gas gaps with the top of the sensor positioned at various distances relative to the midplane, as indicated in the figure legend.

Figure 22. Comparison of IC temperatures at the beginning (Day 1) and end (Day 26) of a HFIR cycle in "core-facing" (upper) vs "reflector-facing" (lower) orientations.

Figure 23. IC temperatures in the WEC pressure sensor at the beginning (Day 1) and end (Day 26) of a HFIR cycle in a "core-facing" orientation. 


\section{LIST OF TABLES}

Table 1. HFIRCON parameters and values used for the neutronics analyses....................................... 21

Table 2. Materials and dimensions used for the neutronics analyses................................................... 22

Table 3. Maximum heat generation rates, including individual contributions from various sources

of heating.

Table 4. WEC temperature sensor model input parameters. 


\section{ACKNOWLEDGMENTS}

This research was sponsored by the Nuclear Science User Facilities Program of the US Department of Energy (DOE) Office of Nuclear Energy. The report was authored by UT-Battelle under Contract No. DE-AC05-00OR22725 with DOE. Kory Linton served as the program manager for this award, which is led by Westinghouse Electric Company (WEC). David Bryant and Bob Sitterson of Oak Ridge National Laboratory (ORNL) assisted with testing of the compression seals. Chelo Chavez and Rocky Estes (ORNL) managed component manufacturing. Chris Bryan, Maureen Searles, Dillon Inabinett and Leonard Mostella (ORNL) provided input on experiment safety, cabling, and future installation in the High Flux Isotope Reactor. 


\begin{abstract}
The ability to deploy new nuclear fuels for current or future reactor concepts requires carefully designed experiments to generate data to support fuel qualification. Ideally these experiments would include stateof-the-art sensing to maximize the amount of in situ data that can be collected during operation. Furthermore, advanced reactor systems can take advantage of integrated in-core sensing technologies to maximize fuel utilization, reduce unnecessary conservativism in design margins, and improve operator's understanding of limiting peaking factors. Before any novel sensing technologies can be readily adopted for nuclear applications, they must first demonstrate acceptable performance in test reactors. This report summarizes the preliminary design and analysis of the most highly instrumented irradiation experiment ever performed in the removable beryllium (RB) positions of the High Flux Isotope Reactor (HFIR) at Oak Ridge National Laboratory (ORNL). The Wireless Instrumented RB Experiment 2021 (WIRE-21) will test a wide range of sensors including wireless sensors being developed by Westinghouse Electric Company (WEC) that could provide in situ measurements of peak fuel temperatures and fuel rod pressurization due to fission gas release. The ability to wirelessly transmit a signal through the fuel rod's cladding is critical to improving fuel monitoring capabilities without requiring signal penetrations through the cladding pressure boundary, which would significantly impact fuel fabrication, handling, and operation. Other sensors that will be tested in WIRE-21 include an array of thermocouples, self-powered neutron detectors (SPNDs), and spatially distributed fiber-optic temperature sensors. More generally, WIRE-21 will establish a flexible irradiation vehicle design to allow accelerated, economical testing of advanced sensor technologies while leveraging the extremely high neutron flux that is available in HFIR.

This report summarizes the mechanical design for WIRE-21, the experimental test matrix, initial neutronic and thermal design analyses, and the active monitoring and control system enhancements necessary to support testing of advanced sensor technologies. The containment for WIRE-21 is similar to previous RB irradiation vehicles but includes a few modifications, most notably the use of integrated compression seals to pass a larger number of sensor leads through the experiment's pressure boundary. In addition to the sensor leads, inert gas lines are passed into the experiment to enable active temperature control and the ability to pneumatically actuate a bellows-driven pressure sensor. WIRE-21 is targeting temperatures $\left(300-350^{\circ} \mathrm{C}\right)$ and neutron fluence levels $\left(\sim 10^{22} \mathrm{n} / \mathrm{cm}^{2}\right)$ relevant to light water reactors (LWRs), but the flexible design of the experiment vehicle allows much higher operating temperatures $\left(>1,100^{\circ} \mathrm{C}\right)$. Neutronic calculations determine the neutron flux conditions as well as the nuclear heating within the experiments. These results are used as inputs to detailed thermal finite element calculations, which are required to evaluate the complex, three-dimensional heat transfer that occurs within WEC's wireless sensor enclosures. Initial results show that the temperatures of the sensors' enclosures and the metal bellows can be operated near the temperature range of LWR coolants and cladding while simultaneously increasing the temperature of a surrogate fuel material to values in the range of 800 $1200^{\circ} \mathrm{C}$ to simulate centerline fuel temperatures during LWR operation.
\end{abstract}




\section{INTRODUCTION}

Nuclear reactors require instrumentation for controlling the fission process, ensuring safe operation of the plant, and to monitor process variables to maximize system efficiency. A wide range of reactor concepts have been demonstrated and safely operated over the past century. However, the industry has been slow to adopt the latest technological advances in instrumentation and control, particularly for in-core instrumentation. One sensing technology that could greatly benefit the nuclear industry is wireless sensors for monitoring fuel conditions during operation. Traditionally the centerline temperatures and internal pressures of fuel rods are only estimated using conservative calculations based on prior experiments performed in test reactors. The ability to monitor these conditions during operation could reduce unnecessary conservatism, potentially allowing higher operating powers. In addition, instrumenting of lead test rods or assemblies containing advanced fuel materials could provide valuable performance data that could be used to accelerate the licensing process for batch reloads of these fuels. Westinghouse Electric Company (WEC) is developing sensors that measure fuel centerline temperature and rod internal pressure and wirelessly transmit the information through the fuel rod's cladding [1-3]. The ability to wirelessly transmit through the fuel rod to a nearby receiver is quite attractive because the wireless transmitters could be integrated into current fuel rods without sensor leads passing through the fuel rod's pressure boundary. This would prevent concerns regarding failures of the instrument seals as well as more complex handling and disposal of fuel rods with sensor leads.

Before WEC's wireless sensor technology can be reliably deployed, the sensors must be tested under representative in-core conditions to the maximum neutron fluence expected over their operational lifetime, which can take many years, depending on the specific test reactor that is used. This poses a great opportunity to leverage the removable beryllium (RB) positions within the High Flux Isotope Reactor (HFIR) at Oak Ridge National Laboratory (ORNL). HFIR provides one of the highest steady-state neutron fluxes in the world [4], which would allow testing these wireless sensors to higher fluences than what could be achieved in a realistic time frame using other facilities. For example, previous testing of WEC's sensors in the Penn State Breazeale Reactor and the Massachusetts Institute of Technology Reactor reached a maximum total neutron fluence of $1.1 \times 10^{21} \mathrm{n} / \mathrm{cm}^{2}[1,5]$, more than 10 times less than the fluence that can be achieved in $\sim 4$ months of irradiation in HFIR. However, to date, instrumented experiments in HFIR's RB positions have largely been focused on using the minimum instrumentation required to quantify environmental conditions during irradiation testing of nuclear fuels or structural materials. Therefore, the facility's data acquisition system and the design of the irradiation vehicle will need to accommodate a large number of sensing leads, particularly for more advanced sensing technologies that require low-noise triaxial cabling or fiber-optics.

This report summarizes the progress that has been made designing the Wireless Instrumented RB Experiment 2021 (WIRE-21) experiment to test WEC's wireless sensor technology in HFIR to accelerate neutron fluence accumulation and move this sensing technology closer to deployment. Two wireless sensors are being tested to measure the temperature of a reactor fuel surrogate and the displacement of a pressurized bellows during irradiation. In addition to WEC's sensors, the experiment also includes a multitude of active and passive instrumentation to measure local and distributed temperatures, as well as neutron flux, spectrum, and fluence over several HFIR cycles. This report summarizes the mechanical design for WIRE-21, the experimental test matrix, initial neutronic and thermal design analyses, and the active monitoring and control system enhancements necessary to support testing of advanced sensor technologies. WEC's sensor technologies have been described previously [1-3,5] but are briefly mentioned in this report to describe how the experiment is being designed to accommodate this specific sensor geometry. The application of this sensing technology to commercial reactors is outside the scope of this work and is further subject to export control and intellectual property restrictions. 


\section{HFIR EXPERIMENTAL FACILITIES}

\subsection{HFIR CORE OVERVIEW}

HFIR is a beryllium-reflected, pressurized, light-water-cooled and moderated flux-trap-type reactor [4]. The core consists of aluminum-clad involute-fuel plates, which currently use highly enriched ${ }^{235} \mathrm{U}$ fuel at a power level of $85 \mathrm{MWt}$. A typical cycle is 25 days. The reactor core, illustrated in Figure 1, consists of two concentric annular regions, each approximately $61 \mathrm{~cm}$ in height. The flux trap is $\sim 12.7 \mathrm{~cm}$ in diameter, and the outer fueled region is $\sim 43.5 \mathrm{~cm}$ in diameter. The fuel region is surrounded by a beryllium annular reflector approximately $30.5 \mathrm{~cm}$ in thickness. The beryllium reflector is surrounded by a water reflector of effectively infinite thickness. In the axial direction, the reactor is reflected by water. The reactor core assembly is contained in a $2.44 \mathrm{~m}$ diameter pressure vessel, which is located in a $5.5 \mathrm{~m}$ cylindrical pool of water. The WIRE-2 1 experiment will be inserted into an RB position located radially just beyond the HFIR fuel, at a radial distance of $27.3 \mathrm{~cm}$ from the center of the core. The experiment is currently intended to be inserted into RB-5A, shown in the lower left portion of the RB in Figure 1.

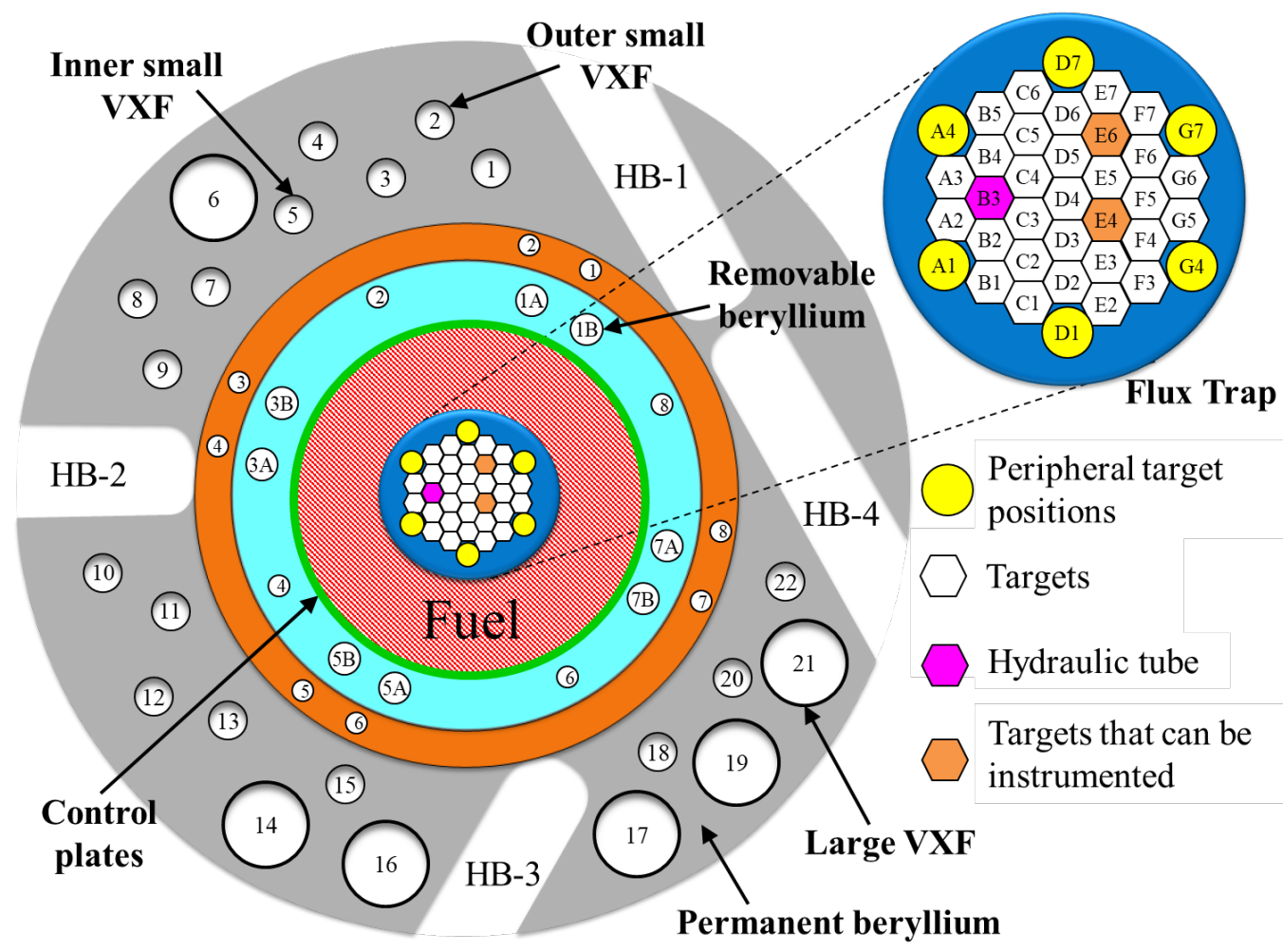

Figure 1. Schematic cross-section of HFIR showing experimental positions.

\subsection{MATERIALS IRRADIATION FACILITY}

The Materials Irradiation Facility (MIF) is located adjacent to the HFIR core and allows active monitoring and control of multiple instrumented irradiation experiments. Its primary purpose is to receive feedback from integrated experiment thermocouples and adjust the flow of various inert gases into each of the spatial regions of an experiment. Gas flow rates are too low to cause significant convective heat removal; instead, gas flows allow online control of the gas composition inside the experiment. Nuclear heating of the internal experiment components must pass to the experiment's outer containment, whose outer surface is exposed to the reactor's primary coolant. Small radial gaps within the experiment create 
resistances to the flow of heat from the experiment's internals. These resistances are carefully controlled by varying the composition, and thus the thermal conductivity, through the gas gaps. Previous experiments have also used the MIF to monitor the release of select fission products during instrumented irradiations of fuels [6-10]. In 2016, modifications to the facility increased the MIF's flexibility, allowing the operation of simultaneous experiments as well as additional instruments through software reconfigurable control modules [11]. A brief summary of the MIF is provided below.

The basic building blocks of the MIF include a gas supply system, gas routing system, effluence system, and a computerized software control system. The gas routing system is software reconfigurable to meet the gas-mixture requirements of specific experiments. The gas supply system is a bank of gas bottles (helium, neon, and argon) connected to a cleanup system to remove oxygen and moisture. The gas routing system controls the pressure, composition, and flow rate of the gas exiting the cleanup system. The software control system uses Allen-Bradley programmable logic controllers (PLCs) to mix as many as three different supply gasses based on measured experiment temperatures, pressures, and flow rates relative to their respective set points. The gas exiting the experiment is routed to the effluence system, which includes a shielded valve box with particulate filters and holdup chambers to allow time for activated argon and other radioactive gases to decay before being vented to HFIR's closed hot off-gas system. The control station computers display the status of the various sensor readings, set points, radiation levels, etc., and include annunciator alarms for alerting the operators and the HFIR control room of any issues. 


\section{WIRE-21 EXPERIMENT DESIGN}

\subsection{WEC WIRELESS SENSORS}

As described previously [1-3], WEC's wireless sensors operate based on the principle of mutual inductance between individual inductors. A section view of WEC's wireless sensor geometries is provided in Figure 2. Within the sensor assembly a combined transmit/receive coil structure is hard wired remotely to electronic circuitry which both energizes the sensor system with a driven signal to a transmit coil and receives the resultant signal from a receive coil pair. The received signal is low in voltage and is amplified and filtered by the electronics for further processing and data acquisition. A signal driven to the transmit coil on the transmit/receive coil structure creates a symmetric magnetic field which in turn energizes an adjacent passive sensor coil structure within the same assembly through magnetic induction. This structure contains both a sensor inductor and a reference inductor which when energized, deliver signals back to the transmit/receive coil through the same magnetic induction process. The only difference between the reference and sensing inductors is that an extension of the sensing inductor's wiring is wrapped around a fuel surrogate material that is designed to operate at a higher temperature during irradiation to simulate light-water reactor (LWR) fuel temperatures. The section of wire in contact with the fuel surrogate acts as a resistance temperature detector (RTD) in measuring the fuel surrogate temperature and affects the amount of signal conveyed by the sensor coil. Both reference and sensing inductors transmit signals wirelessly back to paired inductors in the transmit/receive circuit. The passive circuit does not require any wired connections to the remote transmit/receive electronics.

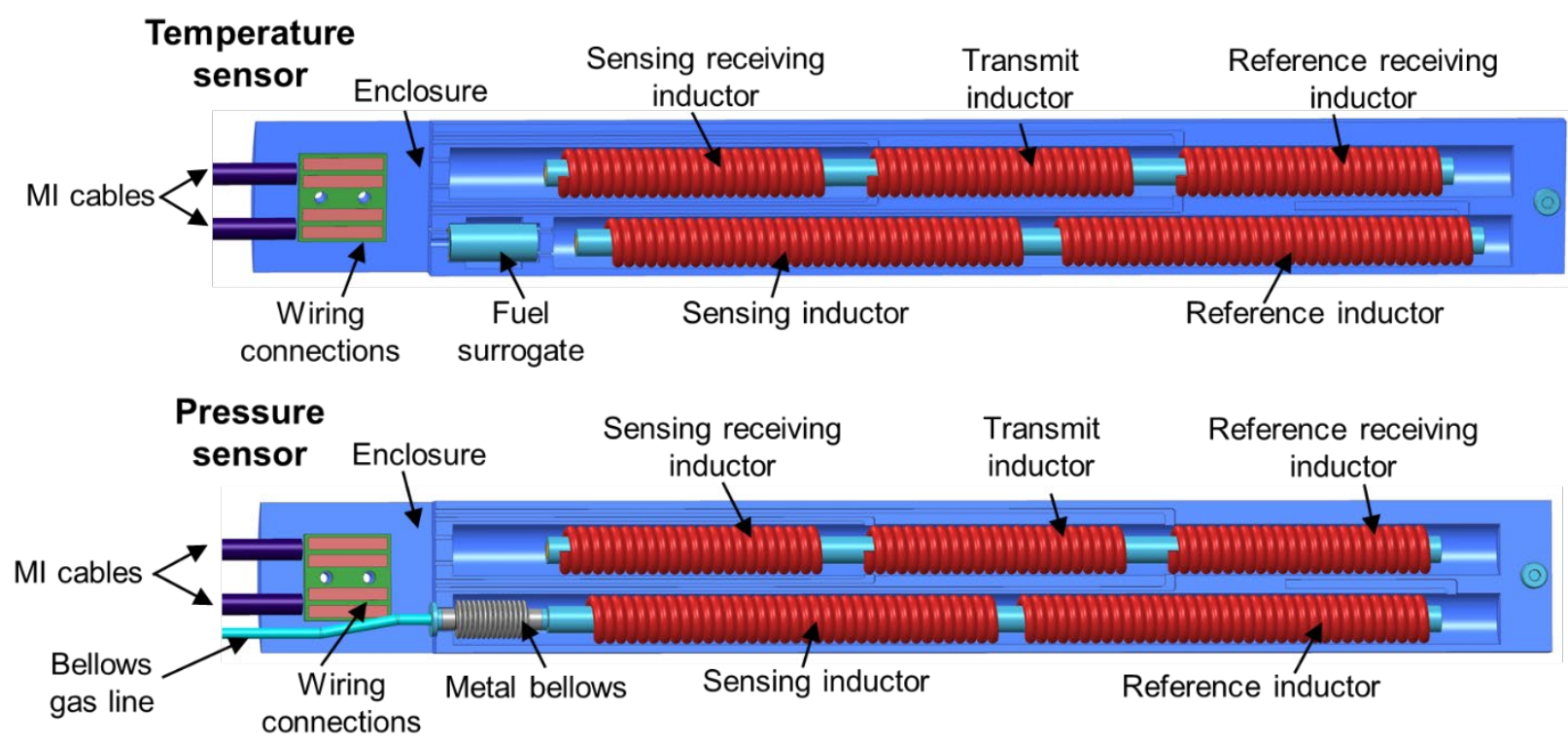

Figure 2. Section views showing WEC's wireless temperature and pressure sensors.

WEC's pressure sensor functions similarly to the wireless temperature sensor with two exceptions: (1) there is no fuel surrogate material but instead a pressurized metal bellows, and (2) the sensing inductor core is attached to the metal bellows and free to traverse axially inside of a wire-wrapped ceramic tube. The ferro-magnetic core is coupled to the end of a metal bellows, which is pneumatically pressurized using a gas supply system in the MIF. Increasing the static pressure supplied to the bellows causes the bellows to expand, which in turn increases the penetration of the core inside the sensing inductor and increases the coupled signal from the sensing inductor to its paired inductor in the transmit/receive circuit. The additional reference coils in the transmit/receive coil structure and the sensor coil structure provide a 
reference signal which is used to compensate for drift or environmentally generated signal changes in the process sensor.

\subsection{WIRE-21 INSTRUMENTATION}

\subsubsection{Wireless Sensors}

As mentioned earlier, the primary goal of WIRE-2 1 is to test WEC's wireless temperature and pressure sensors. In addition, the experiment will contain a wide variety of passive and active instrumentation for measuring temperature and neutron flux during irradiation. Figure 3 shows a schematic detailing the location of each type of active instrumentation. A total of four Mineral Insulated Cables (MIC) will be used to transmit and receive signals from WEC's pressure and temperature sensors (two MICs per sensor), which are located above and below the HFIR midplane, respectively. One MIC traveling to each sensor will contain four electrical leads for receiving signals from the reference and sensing inductors. A second MIC traveling to each wireless sensor includes two electrical leads for powering the transmit inductor and two for an $\mathrm{N}$-type thermocouple for measuring the temperature within the fuel surrogate (for the temperature sensor) and the bellows (for the pressure sensor). 


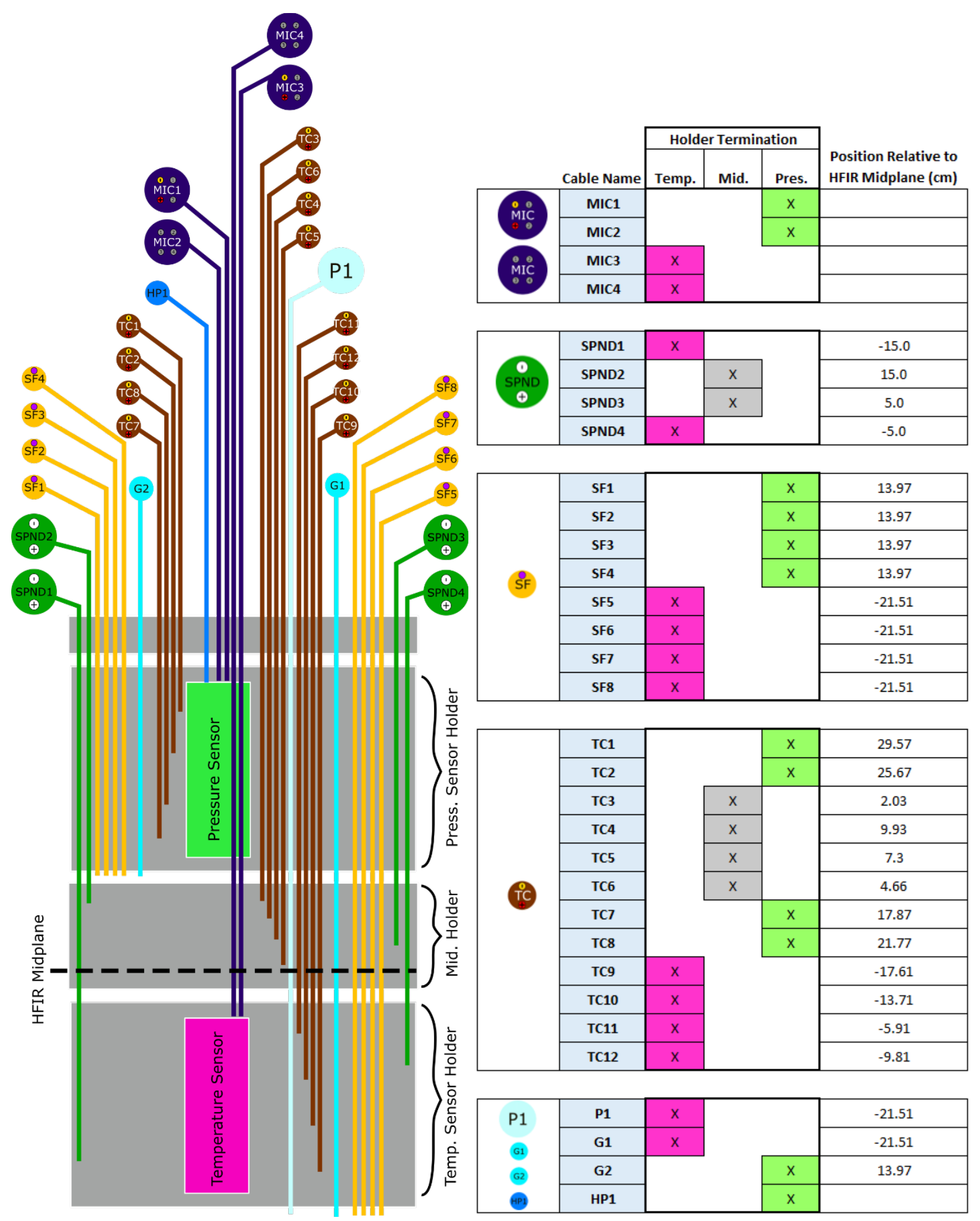

Figure 3. In-core active instrumentation layout for the WIRE-21 experiment (not to scale). 


\subsubsection{Thermocouples and Gas Lines}

In addition to the two thermocouples that terminate inside of WEC's sensor enclosures, 12 stainless steelsheathed, MgO-insulated, N-type thermocouples (labeled TC in Figure 3) will measure local temperature in various regions of the experiment and will provide feedback to the MIF control system. As mentioned previously, temperature control is accomplished by adjusting the composition of gas gaps within the experiment. For this, gas lines (G1 and G2) will be routed below the upper and lower holders containing the pressure and temperature sensors, respectively. A larger diameter helium purge line (P1) is also routed to the bottom of the experiment to allow the experiment to be rapidly flooded with helium if unexpected high temperatures are measured. An effluent gas line (not shown) is located at the top of the experiment's containment and will transfer the supplied gases from the experiment to the MIF's effluence system and ultimately to HFIR's hot off-gas system. A high-pressure gas line (HP1) will provide a static internal pressure within the metal bellows that will be attached to WEC's pressure sensor.

\subsubsection{Fiber-Optic Temperature Sensors}

The included thermocouples will provide accurate measurements of temperature at up to four discrete locations within each holder and provide feedback to the MIF temperature control system. In addition, eight spatially distributed fused silica fiber-optic temperature sensors (denoted as silica fibers, or SF) will be used to provide a more detailed temperature mapping along the experiment length. Optical fibers are an attractive option for temperature-sensing applications due to their small size and ability to provide spatially distributed (millimeter-scale) measurements over tens of meters along a single optical fiber. The fibers to be tested are single mode, operating near $1550 \mathrm{~nm}$, with either a pure silica or fluorine-doped silica core (typically $8-9 \mu \mathrm{m}$ diameter) and a $125 \mu \mathrm{m}$ diameter fluorine-doped cladding. Some of these fibers will include Bragg gratings [12] inscribed with either an ultraviolet continuous wave laser or a pulsed femtosecond laser to provide strong reflections for analyzing the backscattered light signal. Whether the reflections originate from intentional Bragg gratings or from Rayleigh backscatter from random density fluctuations in the amorphous structure, distributed temperature measurements are made by analyzing these reflected signals using optical frequency domain reflectometry (OFDR) $[13,14]$. While the fiber-optic sensors may suffer from radiation-induced attenuation after prolonged neutron exposure $[15,16]$, they should provide accurate temperature distributions during the early phase of the irradiation to inform the temperature gradients within the sensor enclosure.

\subsubsection{Self-Powered Neutron Detectors}

A total of four self-powered neutron detectors (SPNDs) will be included in the WIRE-21 experiment to measure the real-time neutron flux distribution within the experiment. SPNDs consist of two concentric electrodes: a conductive outer sheath (collector) and a neutron-sensitive wire (emitter) that emits a beta particle (electron) upon neutron capture [17]. An insulating material separates the collector and emitter and a radial electric field develops that resists electron flow. When deployed in a reactor, neutron bombardment of the emitter generates sufficiently high-energy electrons to overcome the electric field, resulting in a small electrical current $\left(\mathrm{A}_{1}\right)$ between the emitter and collector. An electrical diagram for the SPND is shown in Figure 4. WIRE-21 will use vanadium as the emitter material, and Inconel 600 for the collector outer sheath as well as the lead wire that is joined to the emitter. A second Inconel 600 electrical lead will be included inside the sheath without an attached emitter to compensate for the current $\left(\mathrm{A}_{2}\right)$ generated from gamma interactions along the length of the SPND. A differential measurement of the currents from the two emitters results in a DC current proportional to the local neutron flux alone. The SPNDs transition to MICs inside the experiment region. Electromechanical devices such as pumps and valves operating near the reactor generate noise, which can couple into nearby electrical detectors. Due to 
the relatively small current produced by SPNDs (typically on the order of picoamps to nanoamps), special care must be taken to mitigate the effects of noise on the measurements.

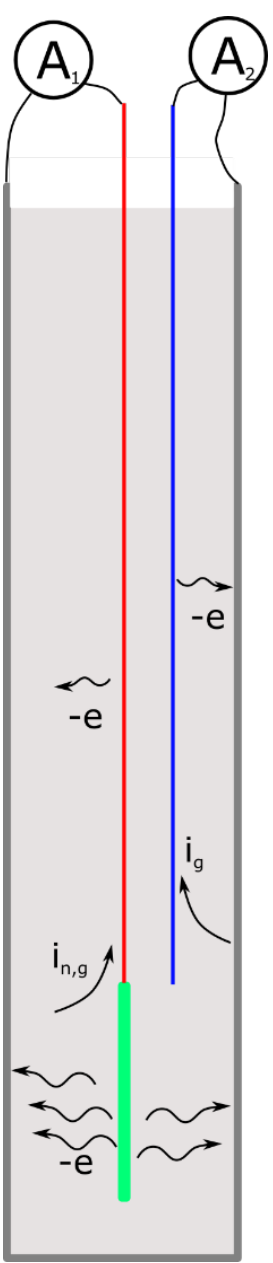

Figure 4. Electrical schematic for the SPNDs that will be used for real-time neutron flux monitoring.

\subsubsection{Passive Instrumentation}

In addition to the previously mentioned active instrumentation, passive instrumentation for neutron flux and average temperature will be included in WIRE-21. Small, sealed, vanadium capsules containing highpurity $\mathrm{Fe}, \mathrm{Ti}, \mathrm{Nb}, \mathrm{Cu}$, and $\mathrm{Co}-\mathrm{Al}$ flux activation wires will be placed below each SPND. Post-irradiation gamma spectroscopy measurements of these wires will be used as inputs to neutron energy spectrum deconvolution codes to determine total neutron fluence levels and neutron flux energy spectra at various locations within the experiment. These measurements will also provide calibration data for the SPNDs in WIRE-21. SiC thermometry, shown as TMPY, TMMY, and TMTY in Figure 6, will also be placed in several locations throughout WIRE-21. Post-irradiation continuous dilatometry measurements will be performed on the $\mathrm{SiC}$ thermometry to determine irradiation temperatures for comparison with measurements obtained from thermocouples and fiber-optic temperature sensors at similar core elevations. 


\subsection{IN-CORE EXPERIMENT REGION}

The in-core region of the WIRE-21 experiment consists of four axial subsections, named for the spacer or holder in that subsection, separated by centering thimbles, or end caps. Figure 5 depicts an elevation view of the in-core region, with major components and positions indicated relative to the HFIR midplane. Moving from top to bottom, these four sections are: Upper Spacer, Upper Holder, Lower Spacer, and Lower Holder. WEC's wireless pressure sensor will be placed inside the Upper Holder, with the top of the Upper Holder coplanar with the top of HFIR's beryllium reflector. The Lower Holder contains WEC's wireless temperature sensor, with the top of the sensor located $5 \mathrm{~cm}$ below the HFIR midplane. The Upper Spacer serves as a guide for the sensor leads and helps dissipate nuclear heating in the leads to prevent them from overheating. The Lower Spacer serves a similar function but also contains four thermocouple junctions (brown leads), two SPNDs (green leads), two flux wire dosimetry capsules (green cylinders below SPNDs), four optical fibers (yellow leads), and 14 SiC passive thermometers (stacked red paralepidids) [18] to provide a more detailed mapping of the temperature and neutron flux distributions near the core midplane.

Cross-sectional views of the Upper Holder, Lower Spacer, and Lower Holder are shown in Figure 6, indicating where the various types of active and passive instrumentation are located. Semitransparent symbols represent instrumentation which pass through the subsection but measure properties in a lower region. The experiment will be oriented so that the radial center of the HFIR core is located to the right of Figure 6. The light green or gray regions in the figure represent the graphite holder or spacer, and the blue regions represents the WEC pressure or temperature sensor. Flux wire dosimetry capsules are located coaxially with and directly below the distal end of each SPND.

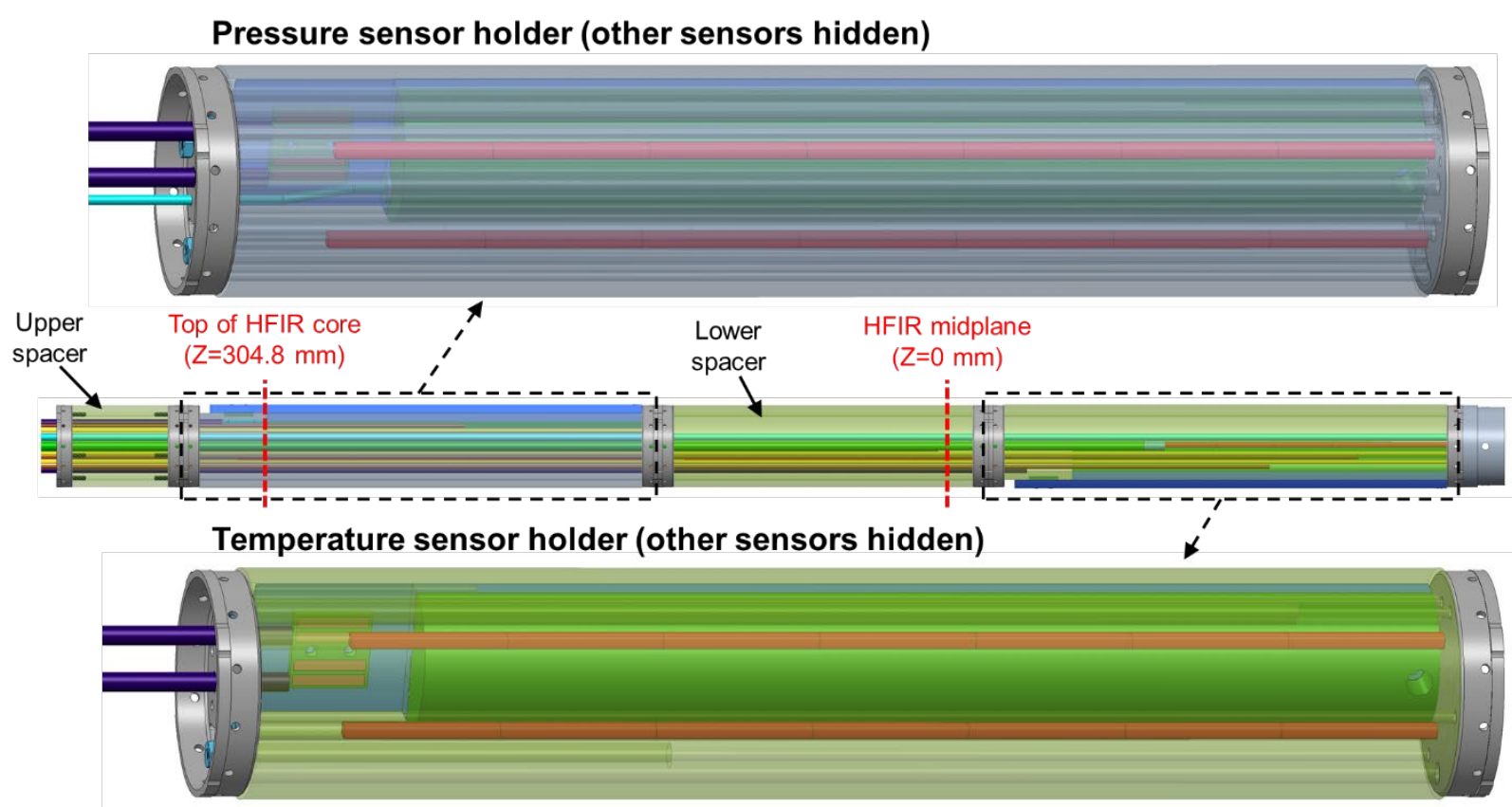

Figure 5. In-core region of the WIRE-21 experiment, with detail views showing the temperature and pressure sensor holders. 


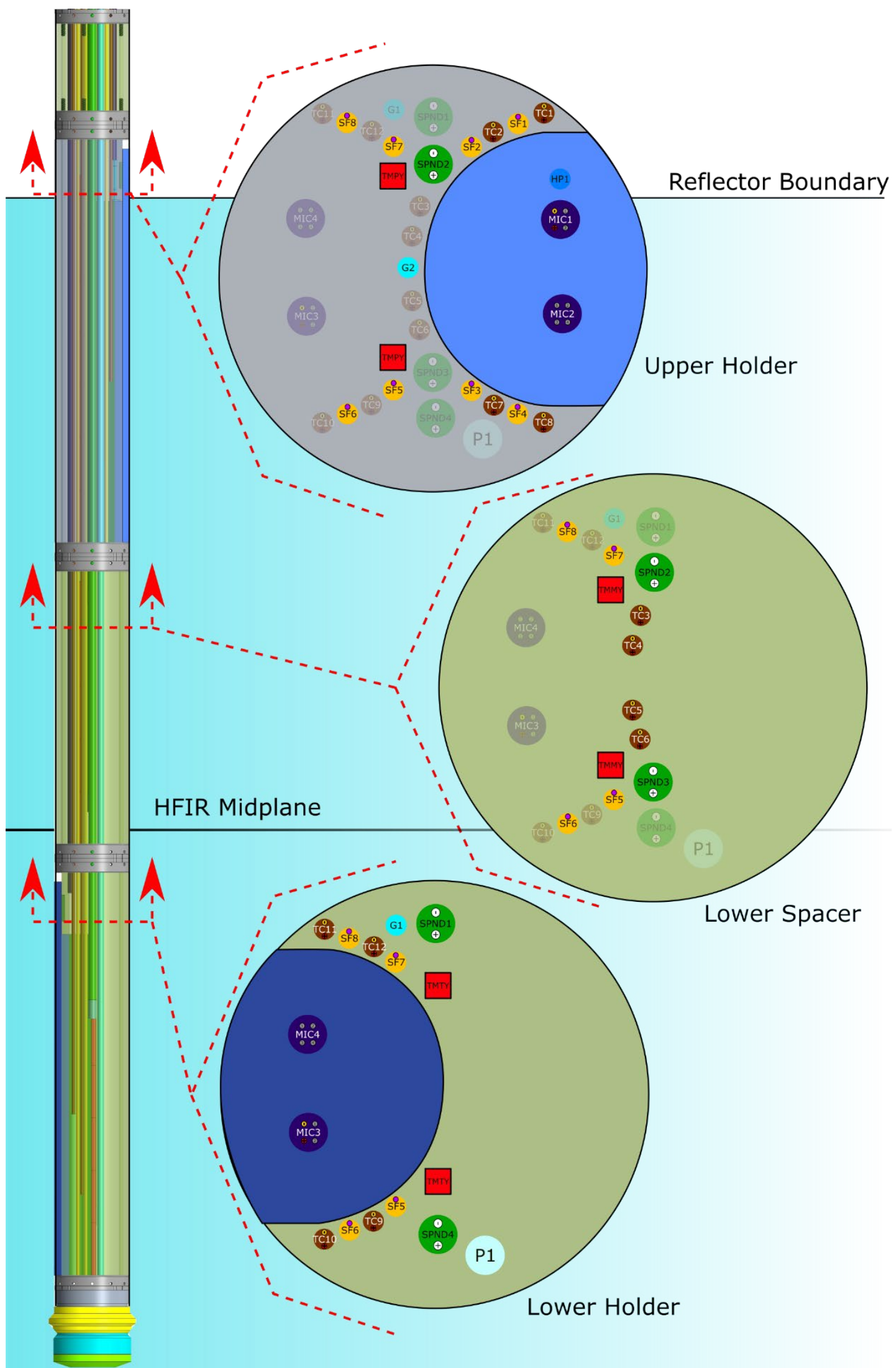

Figure 6. Instrumentation and gas line layout for three primary experiment locations. 


\subsection{EXPERIMENT CONTAINMENT}

The containment for the WIRE-21 experiment is summarized in Figure 7. Most of the experiment is contained within the lower and upper housing tubes, which are welded together using a lower bulkhead adapter. The end of the lower housing tube is welded to an adapter and a rupture disk that will burst if the experiment is unintentionally over-pressurized. Centering tabs keep the experiment centered within the channel of HFIR's RB position, and a welded flow restrictor includes small, machined channels that introduce a sufficiently large flow resistance to prevent the experiment from redirecting too much coolant flow away from HFIR's fuel. The top of the upper housing tube is welded to an upper bulkhead with a machined o-ring groove that allows the experiment to seal against the top lid of HFIR's pressure vessel. Above this seal, lifting lugs allow the experiment to be raised and lowered into the HFIR pool and a welded tag with an engraved identifier helps the operators identify the experiment. Further above these components, a compression seal for the sensor leads is welded to the experiment.

The sensor leads pass through a shield plug, which contains spiral channels that prevent radiation streaming up through the experiment's housing during post-irradiation handling. Figure 8 shows a crosssectional view of all sensor leads and gas lines passing through the spiral channels in the shield plug. The leads continue upward until they reach the compression seal. The compression seal used in this experiment is a 316 stainless steel MHM6 seal from Conax Technologies with a weld neck and a "B" cap, which includes an integrated pipe thread. This seal includes an internal grafoil sealant, which is compressed by six hex head screws as the seal's cap presses against an internal stainless steel follower. When properly torqued, the follower compresses the sealant around the leads, filling the void space and creating a gas-tight seal. A custom gasket is placed between the body and the top cap of the compression seal so that HFIR pool water does not collect inside of the cap. The cap is welded to a hose adapter, which allows a polyurethane hose to be secured using hose clamps to keep the leads dry as they pass up to the top of the HFIR pool.

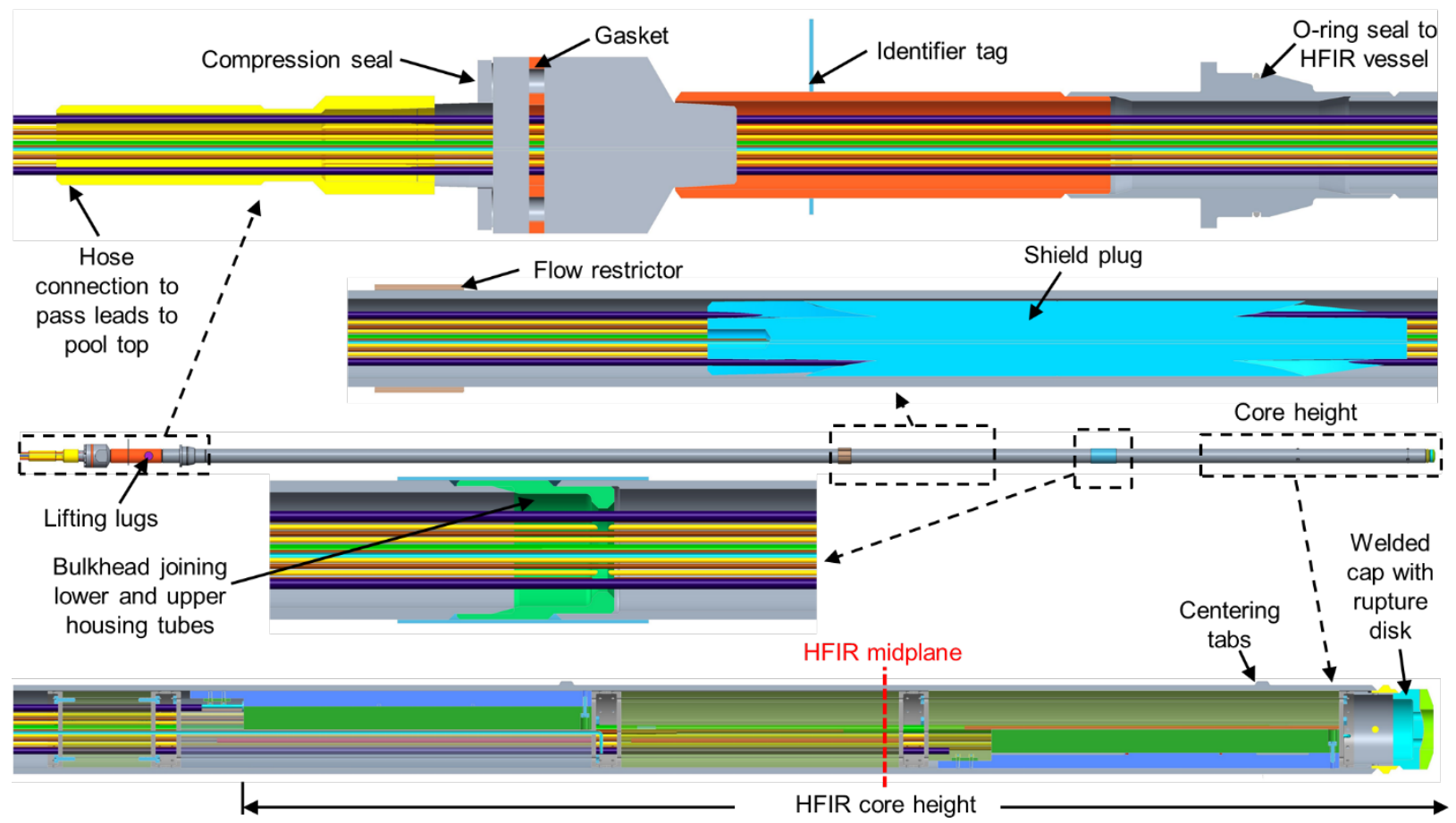

Figure 7. WIRE-21 experiment containment showing the entire length and section views of specific regions. 


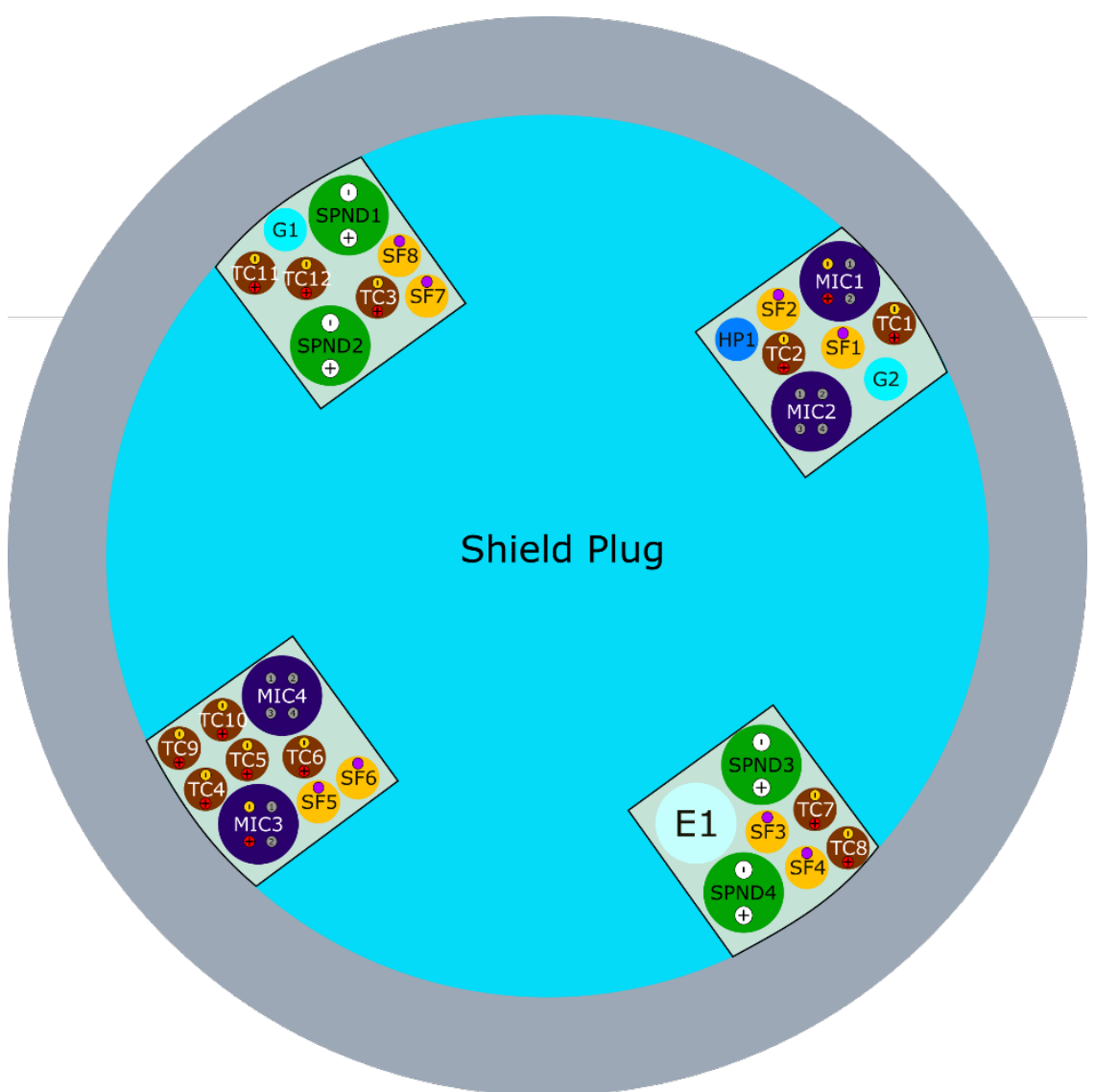

Figure 8. Cross-sectional view of sensor leads and gas lines passing through the spiral channels in the shield plug.

\subsection{HFIR POOL REGION}

A $31.75 \mathrm{~mm}$ (1.25 inch) inner diameter polyurethane flexible hose is used to route the experiment leads from the top of the experiment through the HFIR pool. A Y-adaptor assembly (Figure 9) is used to split the hose to provide a path for gas lines to pass through the reactor pool's wall to the dry wall junction box, which includes permanent gas lines that pass to and from the MIF. All other experiment leads are routed out of the other end of the Y-adaptor in a second polyurethane hose to an experiment-specific junction box, hereafter referred to as the WEC junction box. The $\mathrm{Y}$-adaptor contains two hose fittings for securing the hose with hose clamps, and one compression-style fitting that is attached directly to the drywall junction box. 


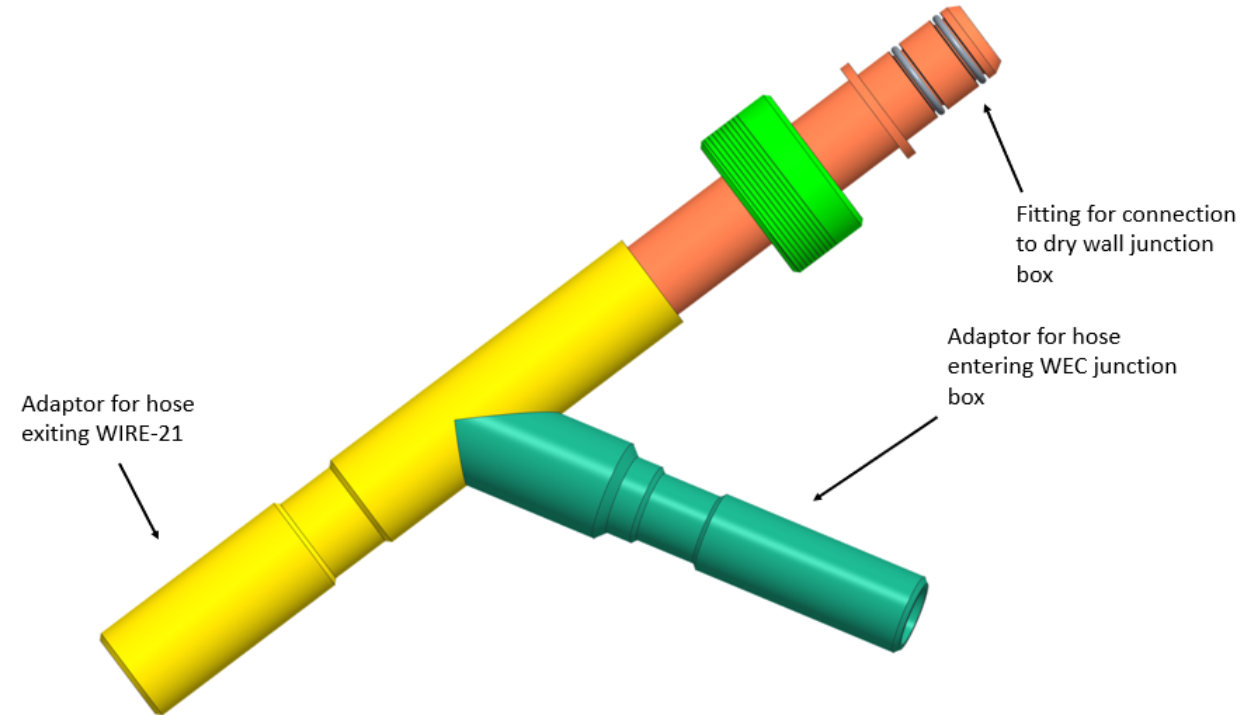

Figure 9. Y-adaptor for splitting the experiment gas lines from the rest of the sensor leads.

The hose exiting the Y-adaptor passes up to the top of the HFIR pool where it is attached to the WEC junction box as shown in Figure 10. This hose attaches to a barbed tube fitting, which is attached via an NPT thread to a tank fitting installed on the wall of the WEC junction box. Each of the various groups of sensors (thermocouples, triax, and fiber optics) are split into separate, smaller hoses (not shown) inside of the WEC junction box and are routed to Amphenol plugs that are connected to mating receptacles inside the electrical junction box. More details regarding the design of the WEC junction box and the routing of sensor leads from the WEC junction box to HFIR's electrical junction box are provided in the next section. 


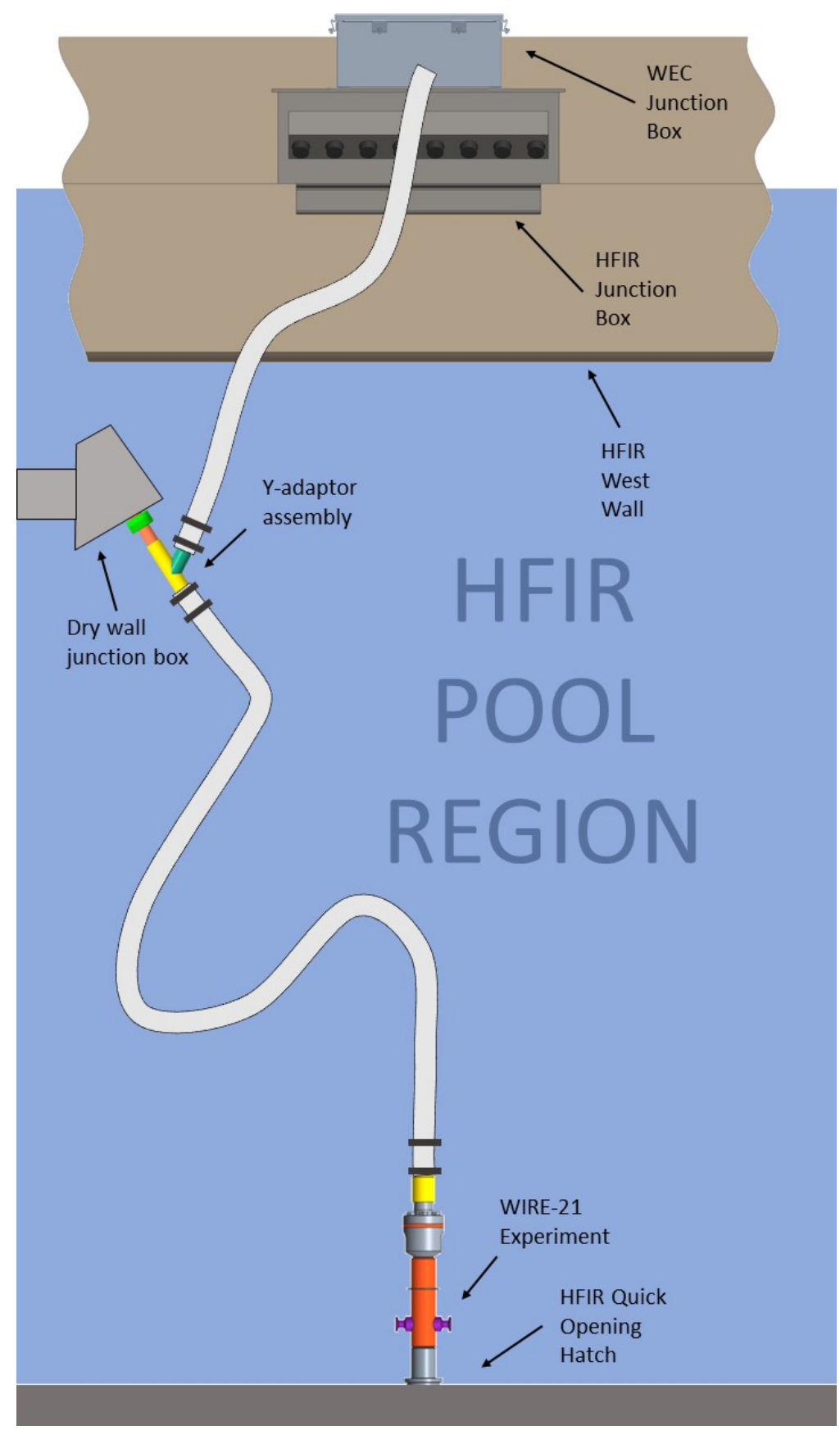

Figure 10. Schematic showing routing of sensor leads within the HFIR pool from the top of the experiment's containment to the electrical junction box.

\subsection{POOLSIDE ELECTRICAL CONNECTIONS}

The sensor leads that pass from the Y-adaptor to the WEC junction box include the MICs for WEC's wireless sensors and the SPNDs, the stainless steel-sheathed Type $\mathrm{N}$ thermocouples, and the fiber-optic temperature sensors. Figure 11 shows a schematic of the cabling that will enter the WEC junction box and the individual outlet hoses that will terminate with Amphenol connectors to mate with corresponding 
Amphenol receptacles in the HFIR electrical junction box. 3D models of the WEC junction box with the tank fittings for the hoses are shown in Figure 12 with and without the cover. Inside the WEC junction box the MICs will transition to flexible triaxial cables (not shown in Figure 12). Similarly, the stainless steel-sheathed thermocouples will transition to flexible thermocouple extension wires. The fiber-optic sensors will transition to jacketed patch cables. A single thermocouple plug will be used for the 12 thermocouples located in the various experiment holders as well as the two thermocouples inside WEC's sensor enclosures. Similarly, all 8 fiber-optic sensors will be coupled to the electrical junction box using a single plug. The MICs for the SPNDs and wireless sensors will be split across two triaxial connector plugs. An additional triaxial pin will be used to provide a direct current power supply for preamplification of the SPND and wireless sensor signals. This preamplification is discussed in more detail later in this section.

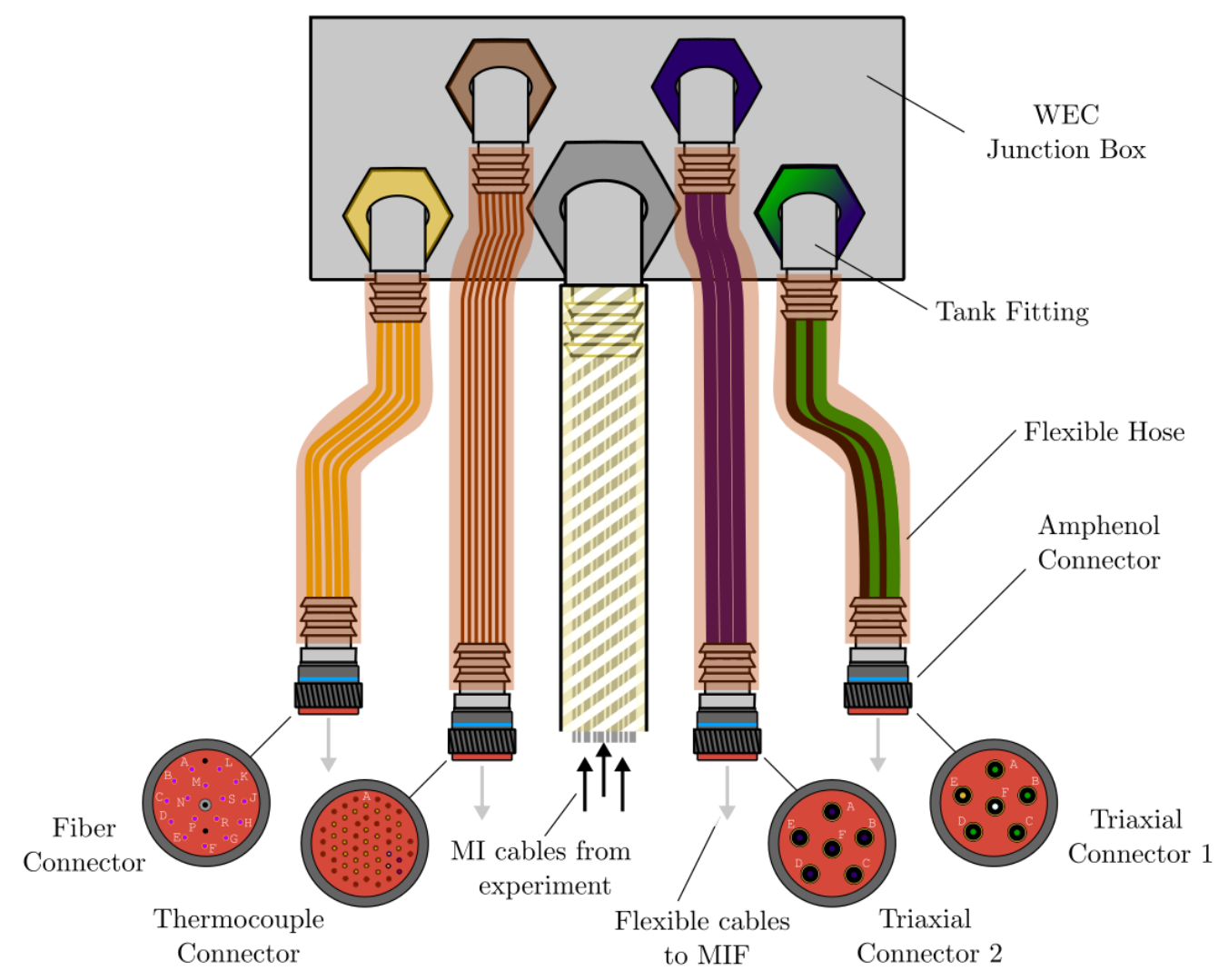

\begin{tabular}{|c|c|c|}
\hline Optical Fibers (8) & Thermocouples (14) & Triaxial Cables (11) \\
\hline - Singlemode Optical Fiber (8) & ¿Sensor Thermocouples (2) & - Preamplifier Power Supply (1 \\
\hline & ¿Ext. Thermocouples (12) & $\begin{array}{l}\text { - SPND (4) } \\
\text { - Experiment Signal (6) }\end{array}$ \\
\hline
\end{tabular}

Figure 11: Cabling schematic for the experiment junction box. 

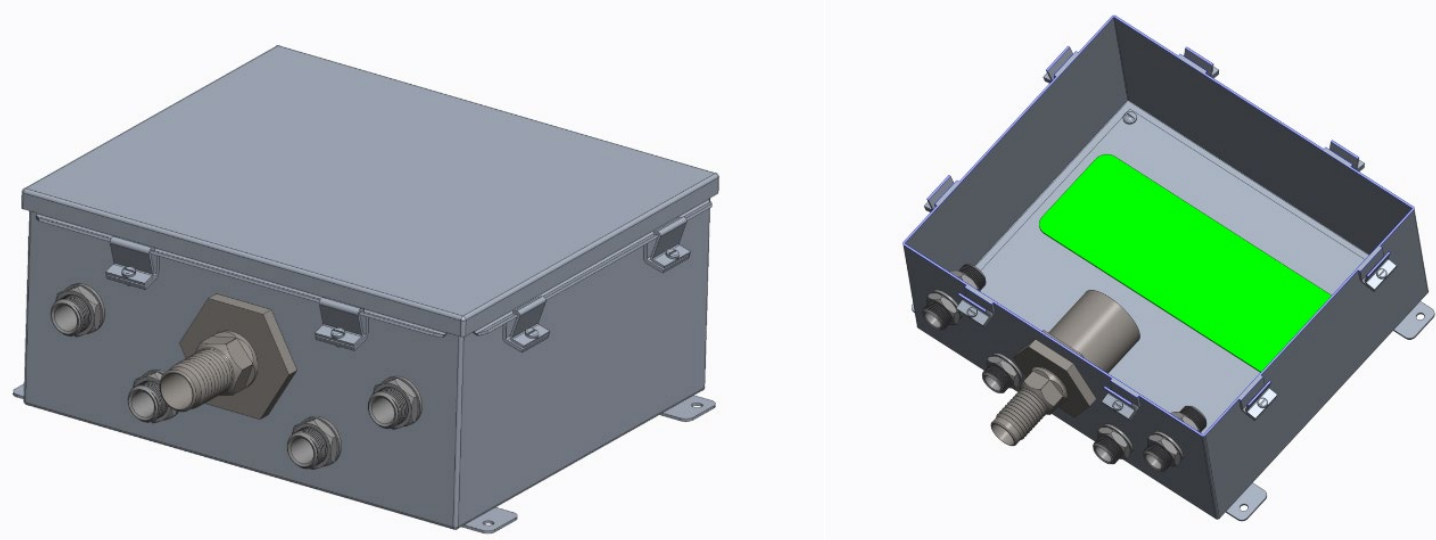

Figure 12: WEC junction box with and without its lid. Internal components are not shown.

\subsubsection{Wireless Sensors}

The drive signal (TX) for the wireless sensors must be transmitted from equipment in the MIF to the transmitting inductors shown in Figure 2. Because the signal must couple wirelessly to and from the sensing and reference inductors, the two inductively coupled output signals - the reference signal (REF) and sensing signal (RX) - have relatively small voltage outputs. Therefore, special provisions are being made to maintain signal integrity over these long lengths. MICs will be run with the outer conductor connected to earth ground in the MIF to provide shielding for the signals carried on the internal conductors. Additionally, preamplification of the RX and REF signals will be performed prior to entering triaxial cables, which will carry these signals from the WEC junction box to the data acquisition systems (DAQs) in the MIF. These preamplifiers use an instrumentation amplifier to reject the common-mode noise present on the RX and REF signals. Jumper connections within the preamplifier circuitry will give the option to bypass the preamplifier for troubleshooting or optimal operation if preferred

\subsubsection{Self-Powered Neutron Detectors}

As the SPNDs pass up through the approximately 4 meter length of the experiment containment, their sheaths will almost certainly be grounded to the experiment housing, which has a great potential for the injection of noise artifacts. To overcome this challenge, two analog front-end (AFE) circuit designs are being considered. The first AFE design involves the use of commercial ammeters with a resolution on the order of a few femtoamperes (Keithley 6482) to convert the direct current (DC) from both emitters to voltages on the order of 5-10 V, which can be recorded by a commercially available DAQ. This system is equivalent to the digital subtraction of two single-ended measurements and suffers from quantization error that propagates through the measurement. The second AFE design first involves the measurement of a voltage across a series resistance into the DC current loops from each of the emitters to the common collector [19]. This voltage can then be amplified by a preamplifier, and a differential voltage measurement can be performed between the two signals using an instrumentation amplifier. While this approach may require additional components to generate an analog differential signal between the two emitters, it does not require that the two signals have the same ground reference, and it dramatically reduces the common mode noise present in each of the signals due to the common collector.

Similar to the MICs for the wireless sensors, the SPND signals will be passed along triaxial cables with their outermost conductor connected to earth ground in the MIF to mitigate noise injection. The SPNDs will also use a preamplifier similar to the MICs for the wireless sensors. This preamplifier will also include analog compensation for gamma interactions. The middle conductor of the triaxial cables will 
carry the signal ground, and the center conductor will carry the signal. In this configuration the earthgrounded outer conductor will shield the internal conductors as the signal is carried from the preamplifier in the WEC junction box to the MIF.

\subsubsection{Thermocouples}

Inside the WEC junction box, the thermocouples will transition to flexible Type $\mathrm{N}$ thermocouple extension wire using a solder-joint. The flexible thermocouple extension wires will be connected to the electrical junction box using copper contacts in an Amphenol-style connector. The transition between Type $\mathrm{N}$ thermocouple wires (Nicrosil/Nisil) and copper could cause concerns regarding dissimilar material connections, the associated effects on the voltage measured in the MIF, and how voltage relates to the true temperature of the thermocouples in the experiment. However, if the junctions involving Nicrosil (or Nisil) and copper are at the same temperature, the induced voltage at these contacts is equal and opposite. Because all connections are made at approximately room temperature, the transitions from thermocouple extension wire to copper contacts in the Amphenol-style connectors are not expected to significantly affect the thermocouple measurements recorded in the MIF. Furthermore, there is precedent for using copper contacts in these connectors in previous experiments.

\subsubsection{Direct Current Power Supply}

The preamplifier circuitry used for the SPNDs and the RX and REF output signals from the wireless sensors require sending DC supply voltages from the MIF to the WEC junction box at the poolside. Noise from the ambient environment will inevitably be injected into these DC voltages along the length of these cables. As such, linear regulators with large decoupling capacitors at their inputs will be used to step down the voltage from $\pm 24 \mathrm{~V}$ as generated at the MIF to $\pm 15 \mathrm{~V}$ at the input of the preamplifier circuitry. The $\pm 24 \mathrm{~V}$ signals will also be routed through the internal conductors of a triaxial cable with the outer conductor connected to earth ground in the MIF to mitigate the addition of noise to the supply voltages during transmission from the MIF. Although the power required by the preamplification circuitry is minimal, current limiting components and fuses in the power supply circuitry will limit risks due to circuit faults if they occur. Additional current limiting can also be provided at the power sources in the MIF.

\subsection{MIF CABLING}

Existing infrastructure within the MIF has historically relied heavily on thermocouples to monitor experiments. Because this experiment involves more advanced instrumentation, modifications are being made to the electrical junction (MIF e-junction) box at the pool-top of HFIR. These modifications also include removal of legacy materials (cables and connectors) and installation of new junction boxes within the MIF. Currently, the MIF e-junction box houses eight Amphenol connectors containing 30 thermocouple twisted-pair wires, as shown in Figure 13. This junction box is under modification to replace five of the thermocouple connectors with fiber-optic and triaxial (triax) Amphenol connectors. The thermocouple cables for each of the five thermocouple connectors are also being replaced with equivalent fiber-optic or triax cables. The cables pass through the pool wall and run through cable trays to the MIF lab, as shown in Figure 14. 


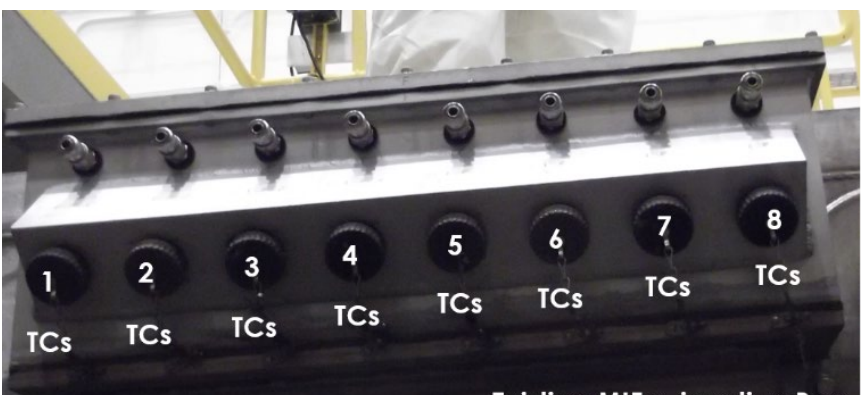

Existing MIF e-junction Box

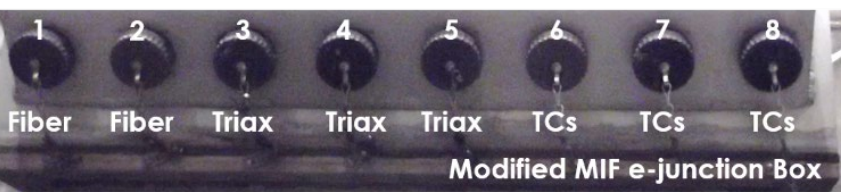

Figure 13. MIF e-junction box modifications.

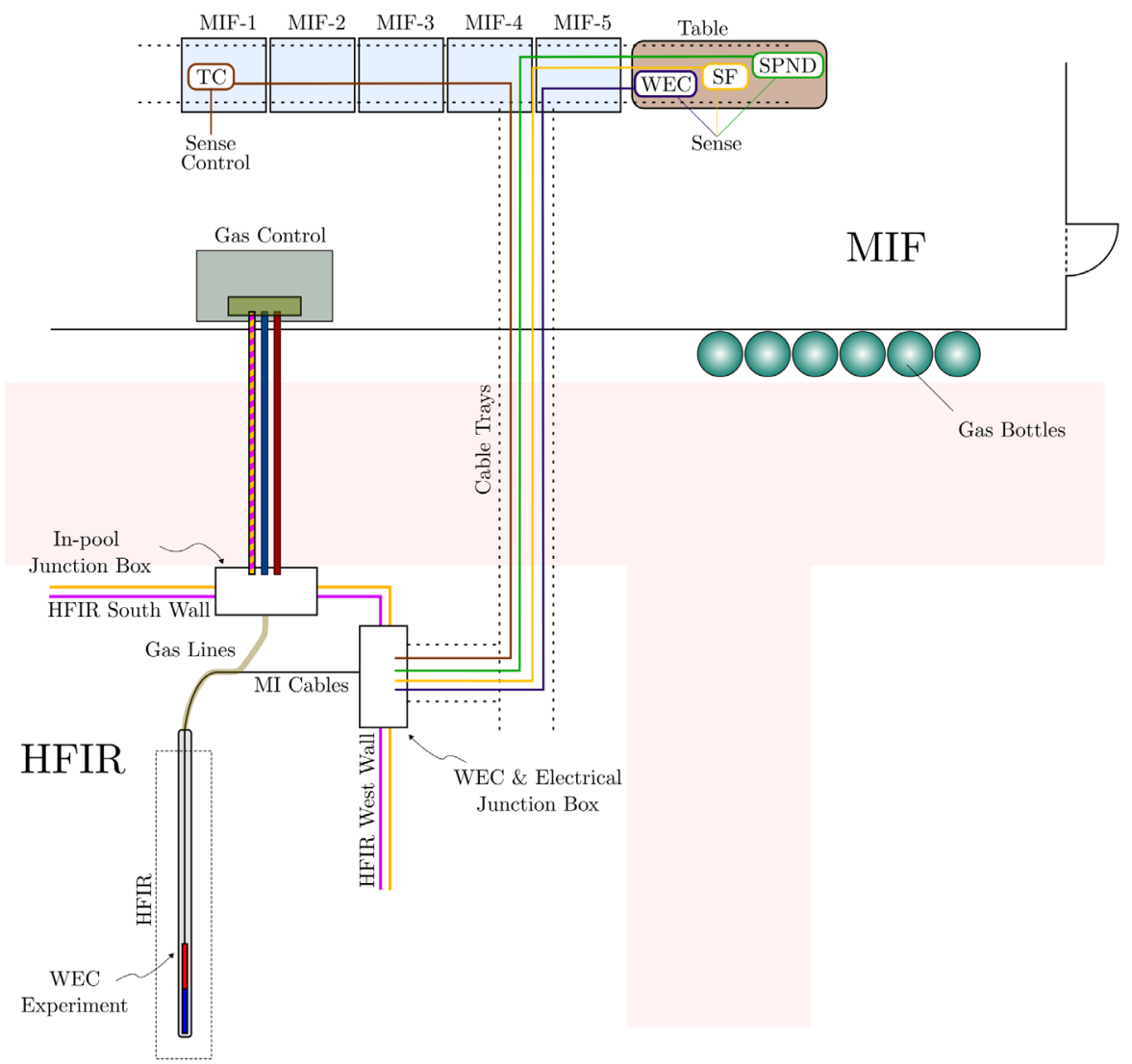

Figure 14. Cable routing between the HFIR pool-top and MIF lab space.

Two new junction boxes are being installed in the MIF to allow easy access for users to connect their DAQs to the experiment. The junction boxes contain connections to the fiber-optic and triax signals coming from the soon-to-be installed receptacles in the MIF e-junction box. As shown in Figure 14, DAQs and other bench-top equipment can be set up on a table located near these junction boxes. Figure 14 shows the bench-top equipment that will be used for WIRE-21, which includes readouts for the wireless sensors (WEC), the fiber-optic sensors (SF), and the SPNDs (SPND). 


\section{MODELING}

\subsection{NEUTRONICS AND DEPLETION MODELING}

\subsubsection{Methods}

Neutronics analyses provide critical information for in-core irradiation experiments including material heat generation rates, displacement damage, transmutation, neutron activation levels, and potential impacts to reactor operations such as reactivity worth and neutron flux tilt. This is especially important in the case of HFIR experiments, as materials are exposed to an extremely high thermal neutron flux on the order of $10^{15} \mathrm{n} / \mathrm{cm}^{2} / \mathrm{s}$ [20]. For the WIRE-21 experiment, the primary goal of the neutronics analysis is to determine the spatial and time dependence of neutron, photon, and decay heating in the experiment materials. Neutron activation must also be estimated to provide input to post-irradiation shipping and handling of the experiment. Neutronics calculations were performed using HFIRCON Version 1.0.5, a code developed at ORNL to perform high-fidelity multicycle target depletion calculations for HFIR experiments. This code couples several preexisting software packages with custom Python scripts and C plugins to automate data transfer between programs. For radiation transport, the ORNL Transformative Neutronics (ORNL-TN) patch for MCNP Version 5.1.60 is used [21]. ORNL-TN was developed to increase MCNP's applicability to ITER neutronics analyses, introduce fixes to bugs present in the MCNP Version 5.1.60 software, and increase its overall efficiency. HFIRCON performs radiation transport calculations for specific experiment geometries in HFIR, depletion calculations based on the results of those calculations, and updates HFIR fuel and experiment material definitions before repeating this process. Various sources of heat generation in the experiment materials including prompt neutron, prompt gamma, and local alpha/beta decay heat are tracked throughout each day of the HFIR cycle, and a followon gamma transport calculation is performed to calculate delayed fission product and local activation gamma decay heating.

HFIRCON also uses the Automated Variance Reduction Generation (ADVANTG) code [22] for generating weight windows and variance reduction parameters, the LAVA Model Interrogator (LAVAMINT) to calculate cell volumes and bounding boxes, and the msx-deplete solver for depletion calculations with ORIGEN [23]. The parameters of a HFIRCON calculation are defined in the controller, a JSON file which dictates the values for cycle length, control cylinder movement, depletion time steps, variance reduction calculation time steps, location of fuel in the MCNP input deck, computational resources, and many others. Table 1 shows some of the various parameters specified for a calculation and the values chosen for this analysis. 
Table 1. HFIRCON parameters and values used for the neutronics analyses.

\begin{tabular}{|c|c|c|}
\hline Parameter & Value & Description \\
\hline initial_decay_time & 0.0 & $\begin{array}{l}\text { Initial decay time in days. This } \\
\text { is relevant for targets that are } \\
\text { irradiated for multiple cycles } \\
\text { and have a decay time between } \\
\text { cycles. }\end{array}$ \\
\hline depletion_step_times & $\begin{array}{c}{[0.0,1.0,3.0,5.0,10.0,15.0} \\
20.0,22.0,24.0,26.0 \\
26.000011574]\end{array}$ & $\begin{array}{c}\text { The time, in days, at which a } \\
\text { depletion step is performed by } \\
\text { ORIGEN via the msx_deplete } \\
\text { solver }\end{array}$ \\
\hline fixed_rod_positions & $\begin{array}{c}{[45.0342,51.1040,51.8000} \\
51.9870,52.7930,54.8030 \\
58.3090,60.3670,63.0200 \\
68.5800]\end{array}$ & $\begin{array}{l}\text { The axial position in } \mathrm{cm} \text { of the } \\
\text { control elements at each } \\
\text { depletion time step }\end{array}$ \\
\hline advantg_steps & {$[0,1,6,8]$} & $\begin{array}{l}\text { HFIRCON iteration steps at } \\
\text { which to perform variance } \\
\text { reduction with ADVANTG }\end{array}$ \\
\hline
\end{tabular}

Other inputs include the platform on which the problem is being solved, the number of processors used to solve the problem, and other resource requirements. For this analysis, the calculations for a single HFIR cycle were performed on ORNL's high-performance computing cluster Apollo with 8 nodes, 32 processors per node, and a 48-hour wall-time. Tallies calculated by MCNP in HFIRCON were scaled by the neutron source strength, corresponding to a reactor power of $85 \mathrm{MW}, 200.7 \mathrm{MeV}$ per fission, and 2.34 neutrons per fission. At each time step, the control cylinder position can either be specified explicitly in the controller (fixed_rod_positions) or found via an eigenvalue calculation performed by MCNP if the positions are not specified. In the latter case, this position is automatically updated in the MCNP input deck via the control element transforms for the next transport and depletion step. This automation significantly reduces the time required for a full cycle calculation and allows for a more detailed analysis.

\subsubsection{Geometry and Materials}

For this analysis, the simplified WIRE-21 experiment geometry shown in Figure 15 was used within the HFIR Representative Cycle 400 MCNP model [24, 25]. The experiment housing, inductor cores (ICs), SPNDs, MICs, and thermocouples for the experiment were positioned at the edge of the holder closest to the HFIR core and then radially mirrored on the opposite side of the holder. This allowed for calculations of the maximum and minimum nuclear heating rates due to radial self-shielding. Additional analyses were performed to determine the impact of shielding of the reflector-side components from the core-side components, but no significant impact was found. The geometry shown in Figure 15 extended from approximately $-40 \mathrm{~cm}$ to $+60 \mathrm{~cm}$ relative to the midplane of the core and was segmented into $1 \mathrm{~cm}$ tall cells. This axial range was used to allow more flexibility in the axial location of the wireless sensors within the experiment housing. However, it is worth noting that MCNP models of HFIR are not very detailed above the reflector regions and therefore may not be perfectly representative. The materials and dimensions used for each of the sensor components are summarized in Table 2. Detailed composition data used in the model are available upon request. 


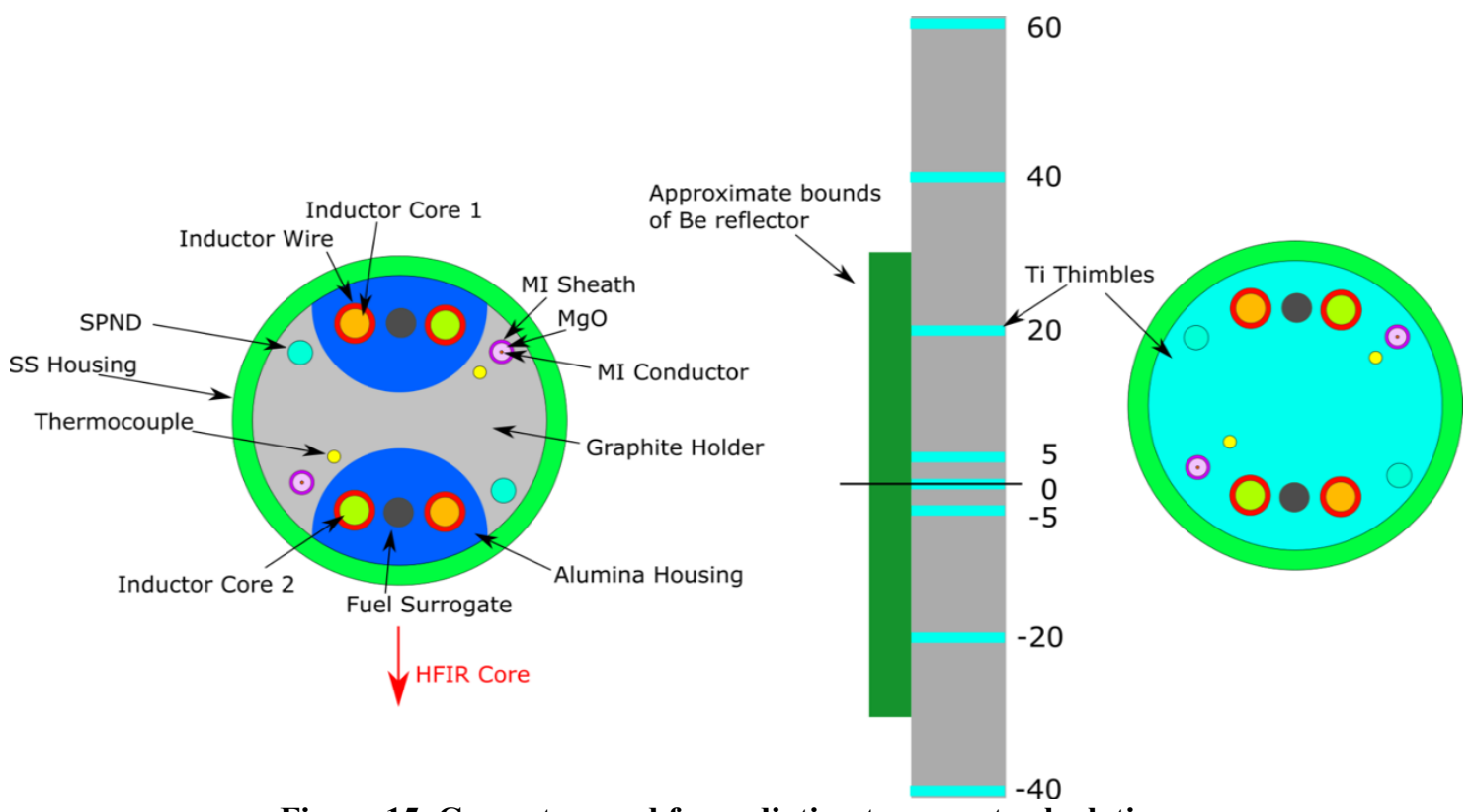

Figure 15. Geometry used for radiation transport calculations.

Table 2. Materials and dimensions used for the neutronics analyses.

\begin{tabular}{|c|c|c|c|c|}
\hline Part Name & Material & $\begin{array}{c}\text { Outer Radius } \\
(\mathrm{cm})\end{array}$ & $\begin{array}{c}\text { Inner Radius } \\
(\mathrm{cm})\end{array}$ & $\begin{array}{c}\text { Thickness } \\
(\mathrm{cm})\end{array}$ \\
\hline Housing & Stainless Steel 304 & 2.11 & 1.8565 & - \\
\hline Holder & Graphite & 1.8565 & - & - \\
\hline Inductor Core 1 & Nickel-Zinc Ferrite & 0.1905 & - & - \\
\hline Inductor Core 2 & Iron & 0.1905 & - & - \\
\hline Inductor Wire & Nickel & 0.262 & 0.1905 & - \\
\hline Sensor Enclosure & Alumina & 1.1075 & - & - \\
\hline MIC Sheath & Stainless Steel 304 & 0.1585 & 0.118 & - \\
\hline MIC Wires & Copper & 0.0254 & - & - \\
\hline SPNDs & Stainless Steel 304 & 0.1585 & - & - \\
\hline $\begin{array}{c}\text { Thermocouple } \\
\text { Sheath }\end{array}$ & Stainless Steel 304 & 0.085 & - & - \\
\hline $\begin{array}{c}\text { Fuel Surrogate } \\
\text { Centering } \\
\text { Thimble }\end{array}$ & Tungsten & 0.1905 & - & 0.2 \\
\hline
\end{tabular}

\subsubsection{Results}

Table 3 shows the maximum heat generation rates for each of the materials listed in Table 2, considering all parts, axial heights, and days in the cycle. Table 3 includes the total heat generation rates as well as the individual contributions from prompt neutrons, prompt photons, delayed photons, and decay heat from photons, alpha particles, and beta particles. Delayed photon heating is only reported at the end of each cycle, but this time corresponds to the maximum photon heating in the RB positions. This is because as the HFIR control plates are withdrawn, the peak fission density in the HFIR fuel moves radially outward, 
which increases the heat generation rates in locations closer to the fuel's outer radial surface. Results are not reported for the relatively thin centering thimbles because their small thicknesses $(0.2 \mathrm{~cm})$ and resulting small volumes lead to high statistical error in the results.

Table 3. Maximum heat generation rates, including individual contributions from various sources of heating.

\begin{tabular}{|c|c|c|c|c|c|}
\hline \multirow{2}{*}{ Material } & \multicolumn{5}{c|}{ Maximum heat generation rates $(\mathrm{W} / \mathrm{g})$} \\
\cline { 2 - 6 } & Prompt neutrons & Prompt photons & Delayed photons & Decay heat & Total \\
\hline Graphite & $1.16 \pm 0.005$ & $6.2 \pm 0.1$ & $1.8 \pm 0.05$ & 0.0 & $9.2 \pm 0.1$ \\
\hline Nickel-Zinc Ferrite & $0.36 \pm 0.005$ & $11.2 \pm 0.6$ & $3.1 \pm 0.3$ & 0.1 & $14.8 \pm 0.7$ \\
\hline Iron & $0.49 \pm 0.007$ & $9.3 \pm 0.6$ & $3.2 \pm 0.2$ & 0.5 & $13.6 \pm 0.7$ \\
\hline Nickel & $0.60 \pm 0.005$ & $12.0 \pm 1.6$ & $3.4 \pm 0.2$ & 0.0 & $16.1 \pm 1.7$ \\
\hline Alumina & $0.59 \pm 0.004$ & $7.9 \pm 0.1$ & $2.3 \pm 0.1$ & 0.3 & $11.0 \pm 0.1$ \\
\hline Stainless Steel 304 & $0.17 \pm 0.002$ & $10.2 \pm 3.9$ & $3.1 \pm 1$ & 0.7 & $14.1 \pm 4$ \\
\hline Copper & $0.06 \pm 0.008$ & $11.2 \pm 7.4$ & $2.9 \pm 0.9$ & 1.3 & $15.5 \pm 7.5$ \\
\hline Tungsten & $0.03 \pm 0.001$ & $18.5 \pm 1.3$ & $5.8 \pm 0.5$ & 0.8 & $25.2 \pm 1.4$ \\
\hline Titanium & $0.11 \pm 0.001$ & $9.4 \pm 0.4$ & $2.6 \pm 0.2$ & 0.0 & $12.1 \pm 0.4$ \\
\hline
\end{tabular}

Relative errors for most heat generation rates are determined from statistical uncertainties from the Monte Carlo calculations performed in MCNP. The exception is the decay heat generation rates, which do not report uncertainties because they were determined using ORIGEN, which is a deterministic code. Because the accuracy of an ORIGEN calculation is dependent on the input neutron flux, the percent uncertainties in the decay heat are expected to be similar to those for the neutron heating results. In any case, the decay heat contributions are small, and the uncertainties are dominated by those for the photon heating. The uncertainties are significantly higher in the copper (MIC wires), tungsten (fuel surrogate), and stainless steel (sensor sheaths) components due to their small cell volumes.

Figure 16 shows how the heat generation rates vary both as a function of axial location and time throughout the HFIR cycle. The heat generation rates steadily increase as the end of the cycle is approached. As mentioned previously, this variation is caused by movements of the HFIR control plates and a radial shift in the peak HFIR fuel fission density. Another interesting phenomenon that was observed in the calculated heat generation rate profiles was the shifting of the axial location for maximum heating further below the core midplane throughout the cycle. The difference in heat generation rates above and below the midplane as the cycle progresses appears to be related to the motion of the control elements in HFIR. In any case, the neutronics analyses provide heat generation rates as a function of material and location for all times throughout the cycle so that detailed thermal calculations - described in the next section - can be performed to estimate the temperature distributions in the WIRE-21 experiment throughout the entire HFIR cycle. 


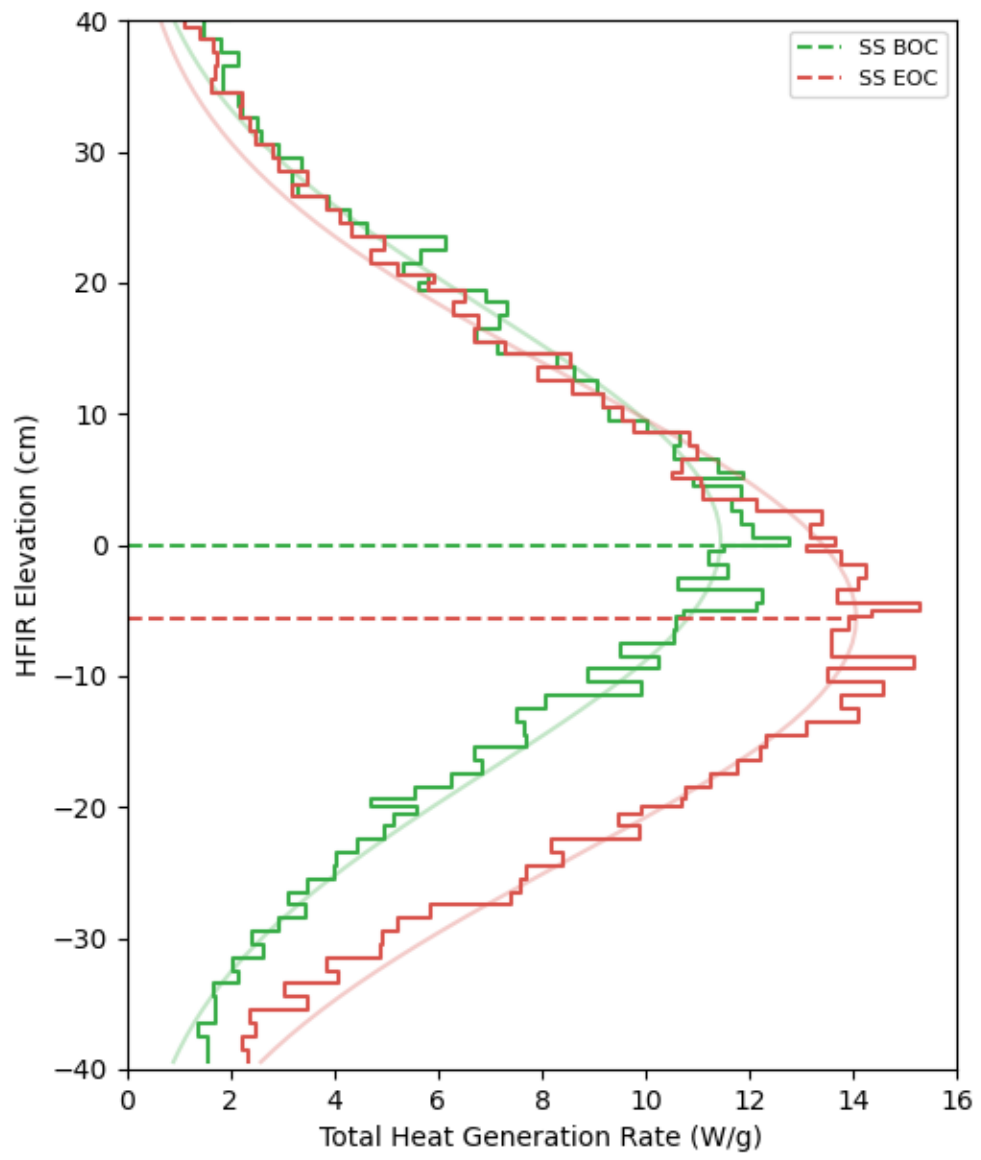

Figure 16. Heat generation rates in stainless steel vs. axial location for various days during a HFIR cycle. Dashed lines represent position of peak heat generation rate for day of cycle.

\subsection{FINITE ELEMENT THERMAL MODELING}

Although WIRE-21 contains many types of passive and active instrumentation, in-pile testing of the prototype sensors developed by WEC is the primary purpose for conducting this experiment. Therefore, achieving optimal neutron flux and temperature conditions for these two sensors were the principal design goals of WIRE-21. Sensors within the experiment assembly were modeled using the ANSYS 2020 R1 finite element analysis (FEA) code for a range of parameterized input conditions, including the geometric dimensions, materials, axial location within the RB position, and several other variables. The purpose of this exercise was to determine the effect of HFIR's cosine-shaped heat generation rate profile on the temperature of the sensors' most critical components: the ICs. The remainder of this section describes finite element modeling efforts to achieve specific design goals for WIRE-21. 


\subsubsection{Geometry}

The WEC wireless temperature and pressure sensors underwent several design iterations over the course of the project based on feedback from irradiation tests of the wireless sensors performed in other reactors and analyses of the various sensor design options performed for this experiment. This required developing FEA models for several different sensor geometries to determine trends in component temperatures. Although the trends reported herein were determined for geometries that differ slightly from the current sensor design described in Section 3.1, they are relevant in explaining the important features of WIRE- 21. Additionally, due to similar internal configurations between WEC's wireless temperature and pressure sensors, the majority of the analyses presented below are specific to the WEC temperature sensor. However, some modeling results are presented for the WEC pressure sensor in Section 4.2.4.

Early CAD models of the wireless temperature sensor provided by WEC included six inductors, as shown in Figure 17, with an arbitrary numbering convention for each inductor. A tungsten "fuel surrogate" (FS) is located above IC 1 to simulate peak fuel temperatures expected from a LWR fuel rod. A stainless steel bellows replaces the FS in the WEC pressure sensor. In a LWR the bellows would be located in the plenum space above the fuel, so it would operate at significantly lower temperatures closer to those of LWR coolants. The inductors were modeled with an iron core, surrounded by tightly wrapped nickel-clad copper wire. All cores were modeled as pure iron for the cases summarized here, although the final sensor may use a silicon iron alloy. In the modeled configuration, inductors 1, 3, 4, and 6 were oriented vertically, while inductors 2 and 5 were oriented normal to the other conductors. The enclosure surrounding the inductors was modeled as $\mathrm{Al}_{2} \mathrm{O}_{3}$, with circular cutouts for each inductor. The outer perimeter of the sensor enclosure is shaped asymmetrically to match the inner radius of the experiment housing. Originally the sensor enclosure was circular and inserted into a cylindrical hole in the graphite holder. However, early results from FEA thermal modeling showed temperatures in the sensors exceeding the Curie point of the inductor material. Therefore, the design was modified to include an asymmetric enclosure to reduce the number of insulating gas gaps between the sensor and outer housing. Wires surrounding IC 2 and IC 5 were modeled explicitly, while wire coils for the remaining inductors were modeled as annuli with an inner and outer diameter equal to the average inner and outer diameter of a double-wrapped helical coil. Gaps between inductors and the sensor housing were also modeled with and without "Resbond," which is a high-temperature, alumina-based adhesive, to determine the impact of filling the insulating gas gaps with a higher thermal conductivity material. 


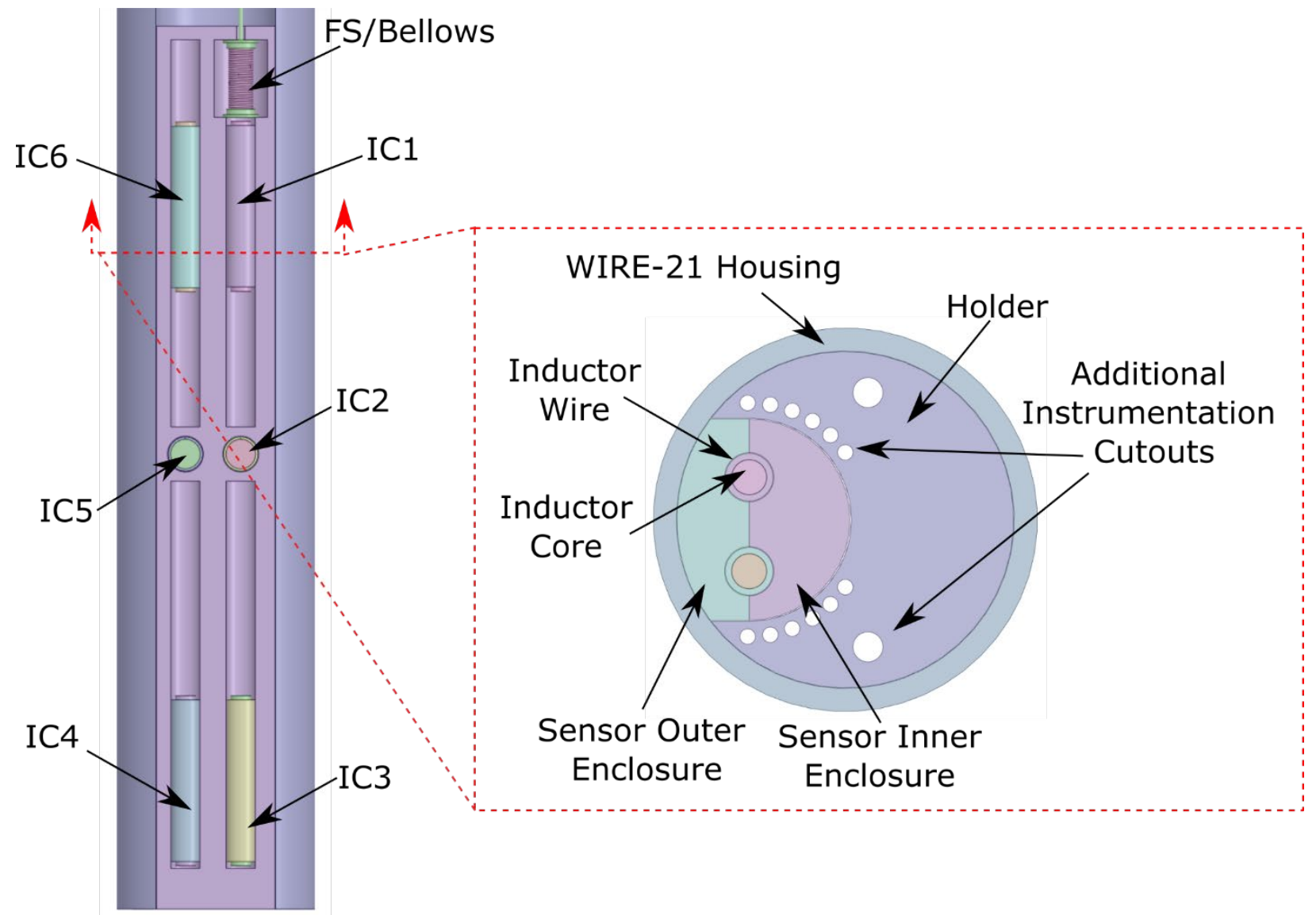

Figure 17. Geometry used for the finite element thermal modeling of WEC's wireless temperature and pressure sensors inside an experiment holder and housing, with numbering conventions for each inductor.

\subsubsection{Conditions}

Several assumptions for experiment conditions were parameterized to evaluate the range of achievable temperatures in the WEC sensors. Table 4 provides a description of these conditions and the range of values used in the FEA models. The majority of these parameters (e.g., geometric dimensions, materials) can be varied during the design phase but become fixed once the design is finalized. However, the helium composition can be controlled in real time using the gas lines that are routed from the MIF. The HFIR cycle day alters the input heat generation rates over the course of the experiment. The power shaping factor also changes over the course of the experiment as the HFIR control plates are withdrawn; however, values included here are meant to represent the extremes of the power profile shape and are intended to bound the temperature spread between sensor components. 
Table 4. WEC temperature sensor model input parameters.

\begin{tabular}{|c|c|c|}
\hline Parameter Name & Description & Range of Values \\
\hline FS OR & $\begin{array}{l}\text { Outer radius of the fuel surrogate, controlling } \\
\text { the gas gap between this component and the } \\
\qquad \mathrm{Al}_{2} \mathrm{O}_{3} \text { enclosure }\end{array}$ & $\begin{array}{c}2.25-2.65 \mathrm{~mm} \\
{[0.24-0.04 \mathrm{~mm} \text { gas gap }]}\end{array}$ \\
\hline Holder OR & $\begin{array}{c}\text { Outer radius of the graphite experiment } \\
\text { holder, controlling the gas gap between this } \\
\text { component and the stainless-steel experiment } \\
\text { housing }\end{array}$ & $\begin{array}{c}18.1-18.5 \mathrm{~mm} \\
{[0.465-0.065 \mathrm{~mm} \text { gas gap }]}\end{array}$ \\
\hline $\begin{array}{c}\text { Power Shaping } \\
\text { Factor }\end{array}$ & $\begin{array}{l}\text { Shaping constant used for the axial heat } \\
\text { generation rate profile in HFIR }\end{array}$ & $\begin{array}{l}\text { Above Mid: } 23.92,30.07 \mathrm{~cm} \\
\text { Below Mid: } 25.25,30.07 \mathrm{~cm}\end{array}$ \\
\hline Percent Helium & $\begin{array}{l}\text { Mole percentage of helium in the } \\
\text { experiment's fill gas, with the balance } \\
\text { composed of argon }\end{array}$ & $50-98 \%$ \\
\hline Holder Material & Flag to indicate the holder material & Graphite, aluminum, titanium \\
\hline Midplane Offset & $\begin{array}{l}\text { Distance between the top of the sensor and } \\
\text { the HFIR midplane }\end{array}$ & $-10-46.9 \mathrm{~cm}$ \\
\hline Resbond Paste & $\begin{array}{c}\text { Flag to indicate whether Resbond paste fills } \\
\text { the gas gaps between the ICs, coils, and } \\
\text { sensor enclosure }\end{array}$ & Exclude/Include \\
\hline HFIR Cycle Day & $\begin{array}{l}\text { Integer indicating which day of the HFIR } \\
\text { cycle is being simulated to scale heat } \\
\text { generation rates }\end{array}$ & $1-26$ \\
\hline Orientation & $\begin{array}{c}\text { Position of sensor within the WIRE-21 } \\
\text { housing }\end{array}$ & Core/reflector facing \\
\hline
\end{tabular}

The goals of the analysis were to determine a set of parameters that would achieve the following conditions:

1. a nominal temperature of $330-350^{\circ} \mathrm{C}$ for ICs $2-6$,

2. a maximum temperature of $500^{\circ} \mathrm{C}$ for ICs $2-6$,

3. a minimum temperature difference of $75^{\circ} \mathrm{C}$ between IC 1 and the other ICs,

4. a nominal temperature of $800-1100^{\circ} \mathrm{C}$ for the $\mathrm{FS}$, and

5. the ability to change temperature via HFIR control plate movement and/or gas composition changes without violating the previous goals.

\subsubsection{Results for Wireless Temperature Sensor}

Modeling emphasis was first placed on analyzing the temperature sensor positioned $5 \mathrm{~cm}$ below the HFIR midplane. Due to the length of the sensor enclosure and the fixed length of the experiment containment, $5 \mathrm{~cm}$ below the midplane was determined as the lowest possible sensor location. Positioning the sensor further below the midplane is advantageous when trying to reduce the heat generation rates to lower the IC temperatures. Multiple thermal calculations were performed using different combinations of FS gas gaps, core power shaping factors, and the presence or absence of Resbond paste surrounding the inductor coils. Although many combinations of experiment conditions listed in Table 4 were modeled, the results presented below used a $0.065 \mathrm{~mm}$ holder-to-housing gas gap, a graphite holder material, and beginning of cycle heat generation rates, unless otherwise specified. The cases presented in Figure 18 were modeled assuming $98 \%$ helium with the sensor positioned $5 \mathrm{~cm}$ below the HFIR midplane. The left-most data point in each of the following plots (Inductor 0) represents temperatures in the fuel surrogate. Error bars 
correspond to the maximum and minimum temperature of each component with the average temperature indicated by squares.

Results indicate that experiment conditions 1 (nominal IC temperatures) and 2 (maximum IC temperatures) are mostly satisfied, although some ICs operate below the $330^{\circ} \mathrm{C}$ threshold. Conditions 3 (IC temperature span) and 4 (FS temperature) are not achieved in any of the cases but come closest in cases with the $0.24 \mathrm{~mm}$ fuel surrogate gap. The temperature spread between the hottest IC (IC 1) and coolest IC (between ICs 2-6) is greater when the smaller power shaping factor is analyzed, as expected. Varying the core peaking factor has the greatest impact on temperatures in ICs 3 and 4, which are farthest from the midplane and experience a steeper gradient in their heat generation rates.

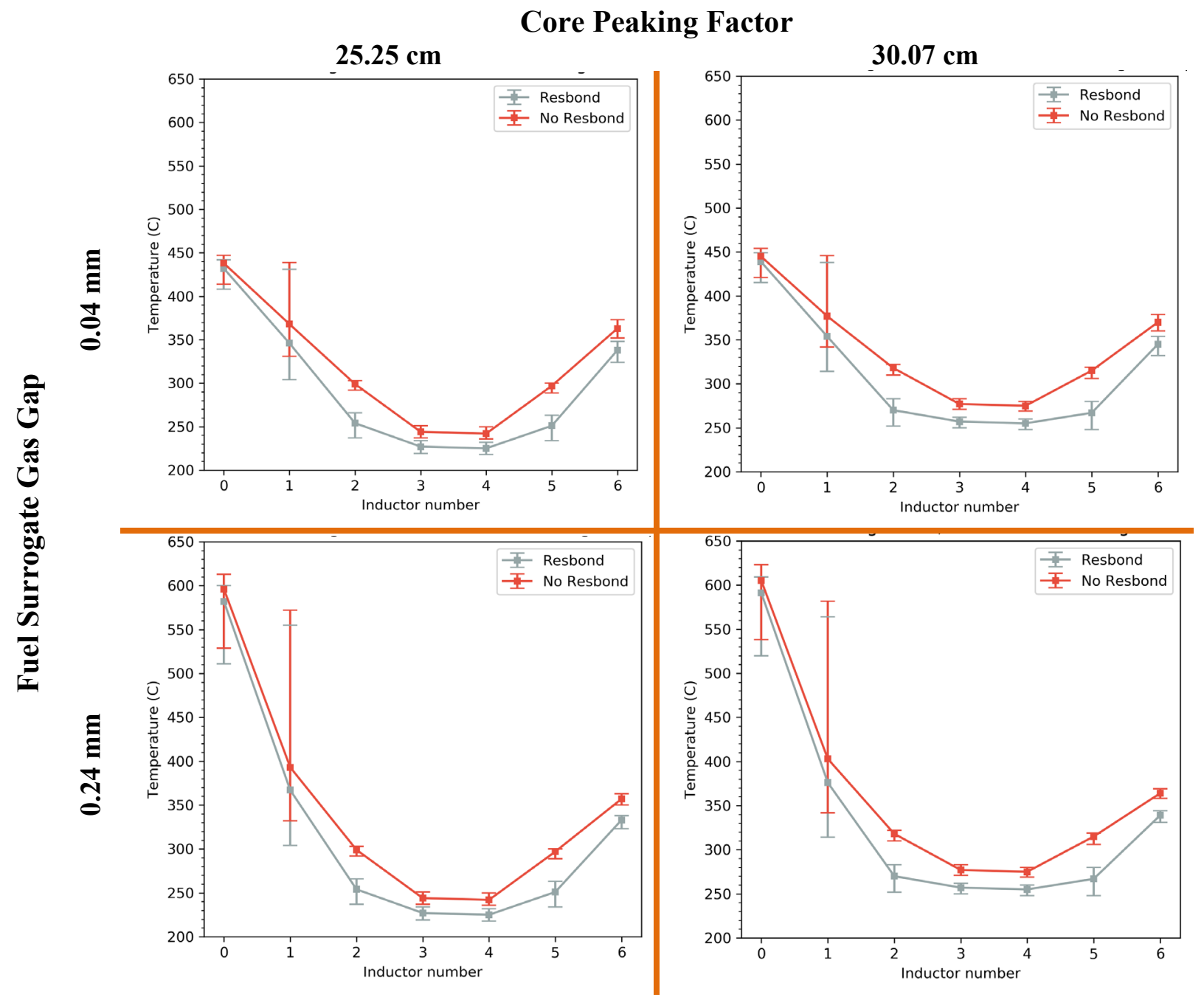

Figure 18. Cases assuming $98 \%$ helium for a range of FS gas gaps and min/max core peaking factors.

Because the $0.24 \mathrm{~mm}$ FS gas gap provided the most promising results, this case was rerun with $75 \%$ and $50 \%$ helium fill gas composition to determine maximum achievable temperatures. Results from these cases (Figure 19) show much higher temperatures in the FS, approaching $940^{\circ} \mathrm{C}$ in the $50 \%$ helium cases. However, the higher FS temperature comes at the cost of higher temperatures in ICs 2-6. 


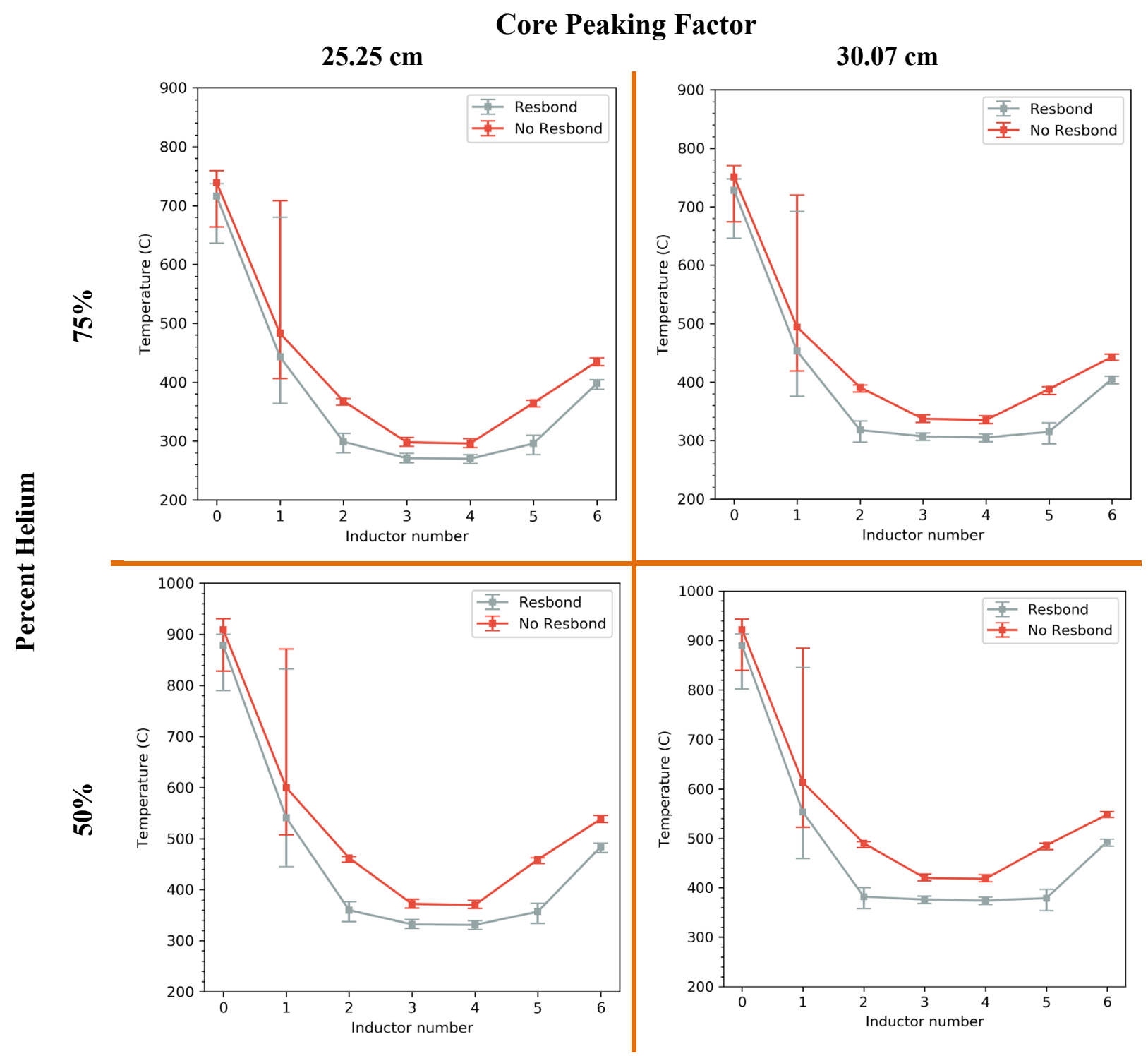

Figure 19. Cases run with $75 \%$ and $50 \%$ helium for a $0.24 \mathrm{~mm}$ FS gas gap and $\mathrm{min} / \mathrm{max}$ core peaking factors.

Although the previous combinations of experiment parameters below the midplane came close to meeting the design goals, WEC expressed interest in potentially testing this sensor at a slightly lower neutron flux. Because there was no space to move the sensor further below the core midplane, additional calculations were performed to evaluate the thermal performance of the temperature sensor above the core midplane, where there is more space to move further away from the core. Three positions above the midplane were analyzed for comparison with results from the sensor located below the midplane, as shown in Figure 20.

The cases with the sensor located above the midplane were initially modeled using the same holder-tohousing gas gaps as the previously reported results. However, temperatures in the sensor components were significantly lower due to the lower heating rates farther from the core midplane (Figure 20). More importantly, the temperature trend between inductors is inverted, with ICs 3 and 4 running much hotter than any of the of the other components, including the fuel surrogate. This can be explained by the core's power profile, which is greatest at the midplane (where IC 3 and IC 4 are closest) and begins to drop off quickly in the vicinity of the fuel surrogate, IC 1, and IC 6. 

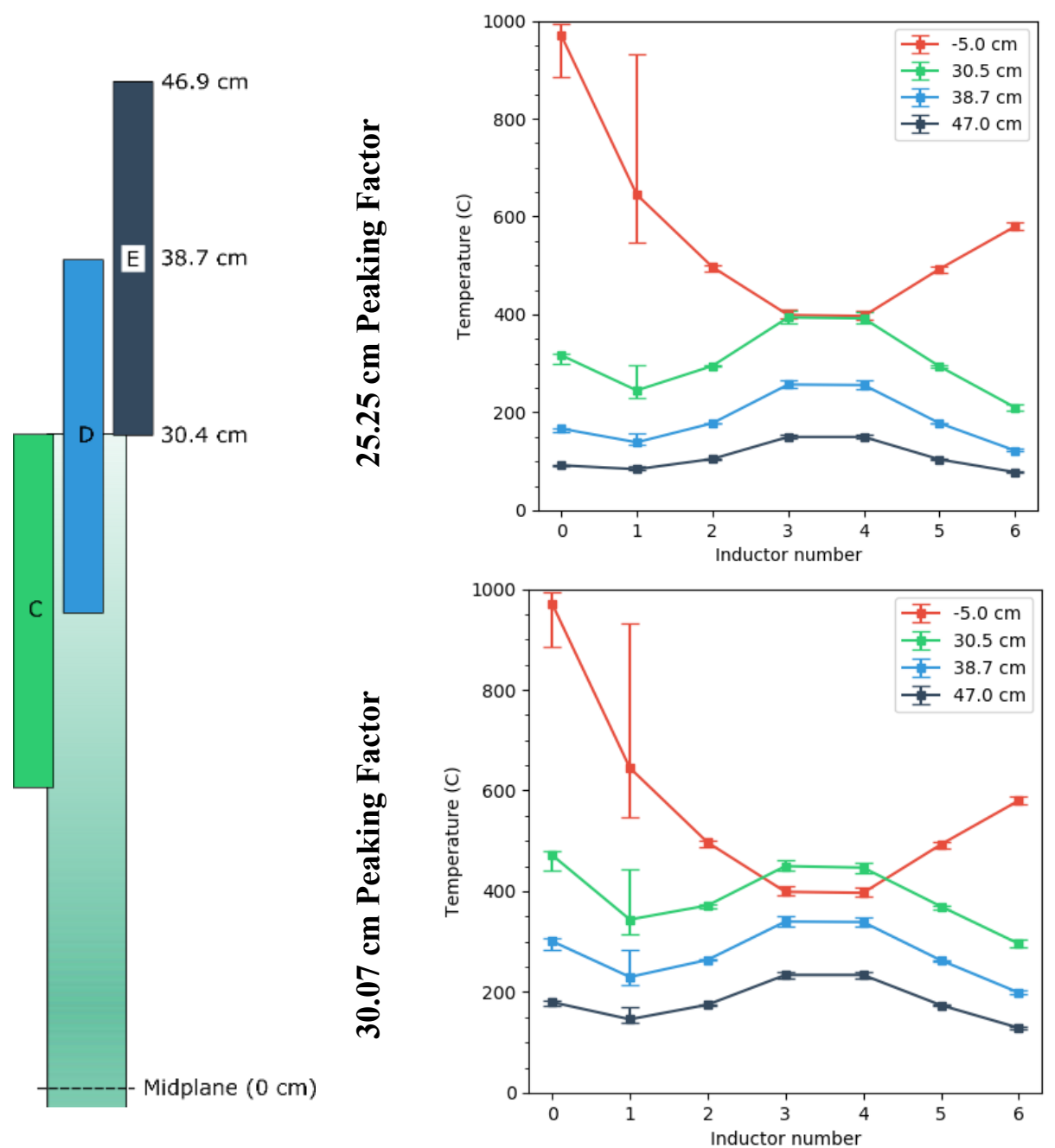

Figure 20. Inductor temperatures for several sensor positions above and below the midplane, with the position of the top of the sensor, relative to the midplane, indicated in the figure legend.

To increase temperatures when positioning the sensor above the core midplane, the gas gap between the holder and housing was increased. Results are shown in Figure 21 for several different holder-to-housing gaps with the sensor located at the top of the midplane (position C in Figure 20). While this does increase temperatures, it also exacerbates the problem of IC 3 and IC 4 being the hottest components in the sensor. Increasing the fuel surrogate gas gap helps to increase the temperature of IC 1; however, positioning the temperature sensor below the midplane was determined to be the most prudent option. 

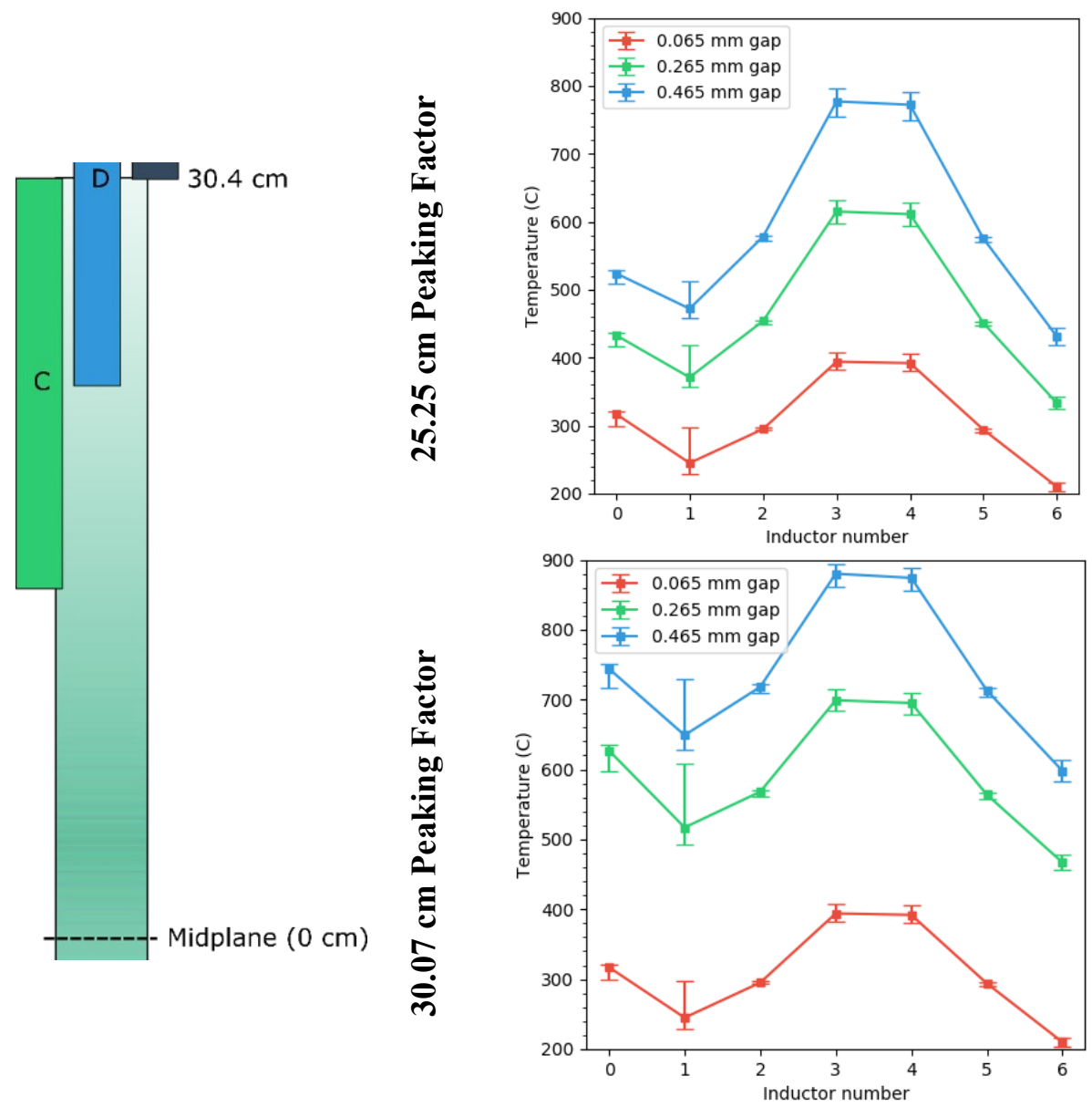

Figure 21. Inductor temperatures for several holder-to-housing gas gaps with the top of the sensor positioned at various distances relative to the midplane, as indicated in the figure legend.

Additional modeling was performed to assess the impact of the WEC sensor in a "core-facing" orientation versus a "reflector-facing" orientation. Depending on the material, components facing the core were found to have $3-7 \%$ greater heat generation rates relative to identical components facing away from the HFIR core. Orientation-specific heat generation rates, as well as time dependent heat generation rates for each day of the HFIR cycle, were applied to FEA models and solved to give the results shown in Figure 22. IC temperatures increased $60-100^{\circ} \mathrm{C}$ from the beginning to end of a HFIR cycle, as material heat generation rates increased with control plate extraction. IC temperatures increased an additional $50-90^{\circ} \mathrm{C}$ when the sensor was oriented to the core-facing position, compared with the reflector-facing position. FS temperatures averaged $1150-1286^{\circ} \mathrm{C}$ with the sensor positioned closest to the HFIR core and 1044-

$1183^{\circ} \mathrm{C}$ in the reflector-facing orientation. However, modifications to the model are being made to more realistically assess axial heat losses from the FS, which will likely decrease the FS temperatures. The current simulations were performed using minimum holder-to-housing gas gaps $(0.065 \mathrm{~mm})$ and $100 \%$ helium fill gas and therefore represent the lowest achievable temperatures of the ICs in the WEC temperature sensor positioned $5 \mathrm{~cm}$ below the core midplane. 

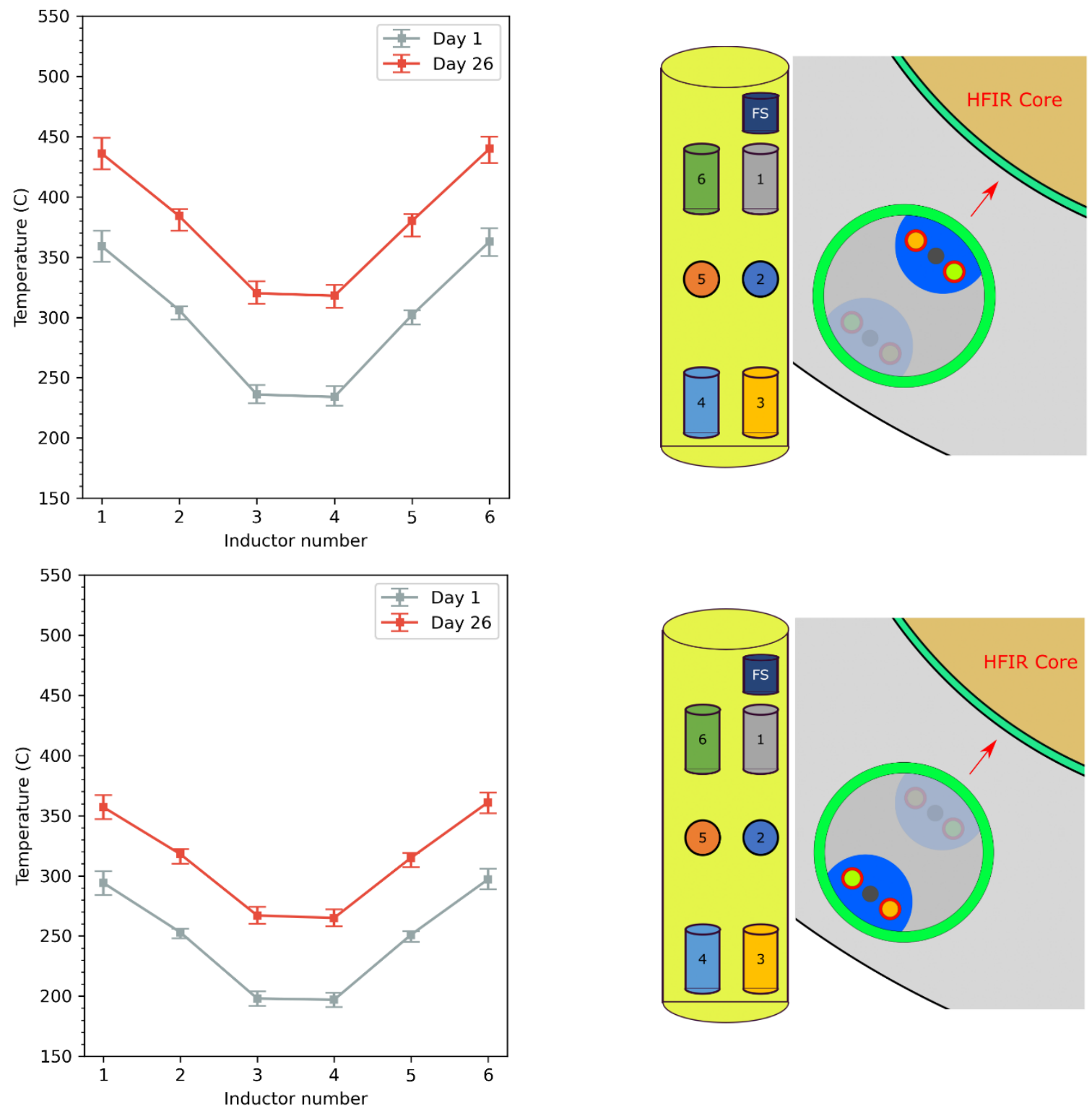

Figure 22. Comparison of IC temperatures at the beginning (Day 1) and end (Day 26) of a HFIR cycle in "core-facing" (upper) vs "reflector-facing" (lower) orientations.

\subsubsection{Results for Wireless Pressure Sensor}

Thermal analyses were also performed for the WEC pressure sensor (Figure 23). For these simulations, the top surface of the pressure sensor was positioned flush with the top surface of HFIR's reflector, which is significantly further from the HFIR midplane compared with the WEC temperature sensor. The ICs showed the highest temperatures in IC 3 and IC 4, and the lowest temperatures in IC 1 and IC 6, which follows the shape of the HFIR power profile. Changes in component temperatures from the beginning to end of cycle are also mitigated in the pressure sensor, due to the smaller change in power profile at the periphery of the core. 

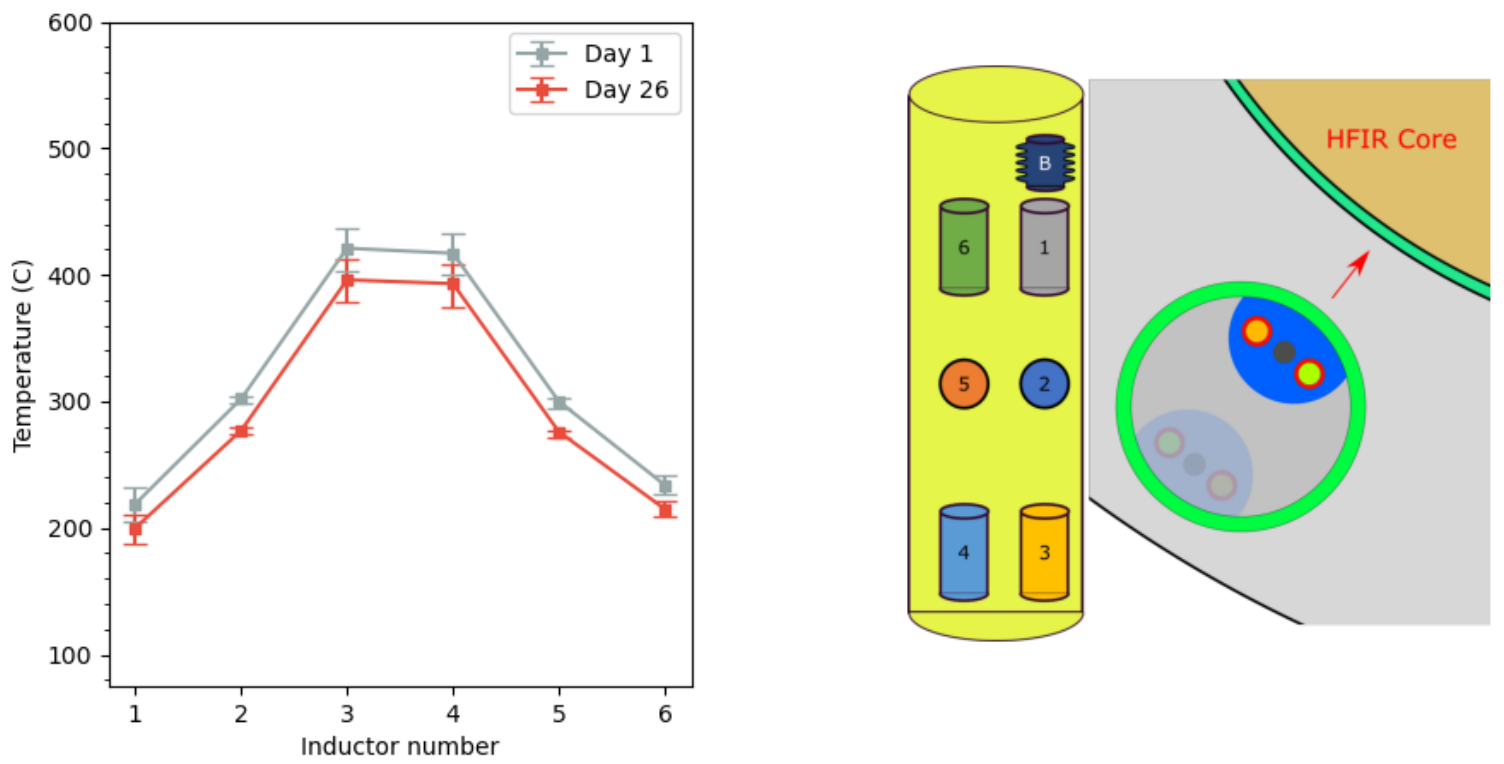

Figure 23. IC temperatures in the WEC pressure sensor at the beginning (Day 1) and end (Day 26) of a HFIR cycle in a "core-facing" orientation. 


\section{SUMMARY AND CONCLUSIONS}

This report summarizes the objectives, design, infrastructure modifications, and analysis supporting a highly instrumented irradiation experiment designed to test WEC's wireless temperature and pressure sensors in HFIR. WEC's wireless sensors are being designed to provide in situ measurements of fuel centerline temperature and rod pressurization. This technology could provide valuable data for qualification of new fuels during irradiation in test reactors. In addition, the wireless sensors could be deployed in operating commercial reactors to provide information to operators to reduce unnecessary conservatisms that are usually applied to fuel performance models, which do not take any credit for online measurements of fuel operating conditions. Before these wireless sensor technologies can be reliably deployed in either test reactors or commercial reactors, they must be tested to end-of-life neutron fluence levels and demonstrate acceptable performance. To this end, the WIRE-21 experiment is being designed to leverage HFIR's high neutron flux to perform accelerated testing of WEC's wireless sensors under accelerated conditions. In addition to WEC's sensors, the experiment is instrumented with many thermocouples, spatially distributed fiber-optic temperature sensors, and passive silicon carbide temperature monitors to provide detailed information regarding the temperature distribution within the experiment. To characterize the neutron flux distribution, four SPNDs are included, along with capsules containing flux wires to evaluate the neutron flux energy spectrum post-irradiation using neutron activation. Finally, gas lines are included inside the experiment to actively control temperature based on the composition of the experiment's fill gas. An additional static gas line is used to actively pressurize WEC's metal bellows-driven pressure sensor. This report summarizes the desired experiment conditions, mechanical design, initial neutronic and thermal design analyses, and the active monitoring and control system enhancements that are being made to accommodate the large number of experiment leads, which include fiber optics and low-noise triaxial cabling. 


\section{REFERENCES}

1. J. Carvajal et al., "Integral Fuel Rod Real-Time Wireless Sensor," in 11th International Conference On Nuclear Plant Instrumentation, Control, and Human-Machine Interface Technologies Orlando, FL (2019) 1000-1012.

2. J. Caravajal et al., Detection Apparatus Usable in a Nuclear Reactor, and Associated Method. US 10811153 B2, U.S.P.a.T. Office, Oct. 20, 2020.

3. J. Caravajal et al., Detection Apparatus Usable in a Nuclear Reactor, and Associated Method. US 10832825 B2, U.S.P.a.T. Office, Nov. 10, 2020.

4. R.D. Cheverton and T.M. Sims, HFIR Core Nuclear Design, ORNL-4621, Oak Ridge National Laboratory, Oak Ridge, TN (1971).

5. J.V. Carvajal et al., "Nuclear Radiation-Tolerant Wireless Transmitter Irradiation Test Results," Nuclear Technology 197 (2017) 201-208.

6. K. Sawa and T. Tobita, "Investigation of Irradiation Behavior of SiC-Coated Fuel Particle at Extended Burnup," Nuclear Technology 142 (2003) 250-259.

7. K. Minato et al., HRB-22 capsule irradiation test for HTGR fuel (JAERI/USDOE collaborative irradiation test), JAERI-Research-98-021, Japan Atomic Energy Research Institute, Japan (1998).

8. F. Homan et al., Irradiation performance of HTGR fuel rods in HFIR experiments HRB-4 and-5, ORNL-5115, Oak Ridge National Laboratory, Oak Ridge, TN (1976).

9. K. Valentine et al., Irradiation performance of HTGR fuel rods in HFIR experiments HRB-7 and8, ORNL-5228, Oak Ridge National Laboratory, Oak Ridge, TN (1977).

10. F. Homan et al., Irradiation performance of HTGR fuel rods in HFIR experiments HRB-11 and12, ORNL-5584, Oak Ridge National Laboratory, Oak Ridge, TN (1980).

11. N.O. Cetiner et al., "An advanced materials irradiation facility for materials and fuels irradiations at the high flux isotope reactor," in Proceedings of Top Fuel 2016, Boise, ID (2016) 389-398.

12. K.O. Hill and G. Meltz, "Fiber Bragg grating technology fundamentals and overview," Journal of Lightwave Technology 15 (1997) 1263-1276.

13. D.C. Sweeney et al., "An Adaptive Reference Scheme to Extend the Functional Range of Optical Backscatter Reflectometry in Extreme Environments," IEEE Sensors Journal 21 (2020) 498-509.

14. M. Froggatt and J. Moore, "High-spatial-resolution distributed strain measurement in optical fiber with Rayleigh scatter," Applied Optics 37 (1998) 1735-1740.

15. C.M. Petrie et al., "High-dose temperature-dependent neutron irradiation effects on the optical transmission and dimensional stability of amorphous fused silica," Journal of Non-Crystalline Solids 525 (2019) 119668.

16. G. Cheymol et al., "High Level Gamma and Neutron Irradiation of Silica Optical Fibers in CEA OSIRIS Nuclear Reactor," IEEE Transactions on Nuclear Science 55 (2008) 2252-2258.

17. H.D. Warren, "Calculational Model for Self-Powered Neutron Detector," Nuclear Science and Engineering 48 (1972) 331-342.

18. K.G. Field et al., "Evaluation of the continuous dilatometer method of silicon carbide thermometry for passive irradiation temperature determination," Nuclear Instruments and Methods in Physics Research Section B: Beam Interactions with Materials and Atoms 445 (2019) 46-56.

19. J.M. Jacob, Industrial control electronics: applications and design. 1988: Prentice Hall.

20. N. Xoubi and R.T. Primm III, Modeling of the High Flux Isotope Reactor Cycle 400, ORNL/TM2004/251, Oak Ridge, TN (2005).

21. S.W. Mosher and S.C. Wilson, "Algorithmic Improvements to MCNP5 for High-Resolution Fusion Neutronics Analyses," Fusion Science and Technology 74 (2018) 263-276.

22. S.W. Mosher et al., ADVANTG-An Automated Variance Reduction Parameter Generator, ORNL/TM-2013/416, Oak Ridge National Laboratory, Oak Ridge, TN (2013). 
23. I.C. Gauld et al., "Isotopic Depletion and Decay Methods and Analysis Capabilities in SCALE," Nuclear Technology 174 (2011) 169-195.

24. G. Ilas et al., Modeling and simulations for the high flux isotope reactor cycle 400, ORNL/TM2015/36, Oak Ridge National Laboratory, Oak Ridge, TN (2015).

25. D. Chandler et al., Modeling and Depletion Simulations for a High Flux Isotope Reactor Cycle with a Representative Experiment Loading, ORNL/TM-2016/23, Oak Ridge National Laboratory, Oak Ridge, TN (2016). 